COMECHINGONIA. Revista de Arqueología. Vol. 25 n 3. 2021. ISSN: 0326-791

\title{
DISPONIBILIDAD Y EXPLOTACIÓN DE ROCAS DE GRANO FINO OSCURAS TIPO POTROK AIKE EN EL INTERFLUVIO GALLEGOS-CHICO (PROVINCIA DE SANTA CRUZ, ARGENTINA)
}

\section{AVAILABILITY AND EXPLOITATION OF DARK FINE GRAINED ROCKS POTROK AIKE TYPE IN THE GALLEGOS-CHICO INTERFLUVE (SANTA CRUZ PROVINCE, ARGENTINA)}

\author{
Natalia A. Cirigliano1, Karen Borrazzo ${ }^{2}$ \\ 1 IMHICIHU-CONICET/FFyL-UBA, Saavedra 15, piso 5, (1083)/Puán 480, (1406), Buenos Aires, \\ Argentina. E-mail: naticirigliano2015@gmail.com \\ ${ }_{2}^{2}$ IMHICIHU-CONICET, Saavedra 15, piso 5, (1083), Buenos Aires, Argentina. \\ E-mail: kborrazzo@yahoo.com.ar
}

Presentado: 04/03/2021

Aceptado: 24/07/2021

\section{Resumen}

En este trabajo se evalúa la explotación de las Rocas de Grano Fino Oscuras tipo Potrok Aike en relación con su disponibilidad en un segmento del interfluvio de los ríos Gallegos y Chico (IGCh), sector central del campo volcánico Pali Aike (CVPA), provincia de Santa Cruz, Argentina. Para ello se analiza mediante muestreos de rocas la abundancia y características de los nódulos de RGFO PKA disponibles naturalmente en el área, así como la distribución y particularidades del conjunto artefactual lítico recuperado en superficie. Los resultados alcanzados muestran diferencias espaciales en la disponibilidad de esta roca, advierten sobre la explotación efectiva de las fuentes secundarias por parte de cazadores-recolectores, indican variabilidad en la intensidad de explotación de nódulos de RGFO PKA en los distintos espacios investigados y sugieren el acarreo antrópico de nódulos a pequeña escala. Los datos obtenidos amplian nuestro conocimiento sobre la disponibilidad y aprovechamiento de la variedad PKA en el CVPA y refuerzan la idea del IGCh como un área clave de captación de esta materia prima como señalaban trabajos previos.

Palabras clave: fuentes líticas secundarias, nódulos, uso del espacio, Patagonia

\begin{abstract}
This paper studies the relationship between the natural availability of dark fine-grained rocks Potrok Aike type (RGFO PKA) and their technological exploitation within a portion of the Gallegos and Chico river interfluve (central Pali Aike Volcanic Field, Santa Cruz province, Argentina). We analyze the natural abundance and attributes of RGFO PKA nodules locally available and assess the distribution and characteristics of archaeological lithic assemblages. The
\end{abstract}


results obtained show: 1) the existence of spatial differences in rock natural availability, 2) the effective exploitation of local sources by hunter-gatherers, 3) variable intensity in quarrying activities across the study area sources, and 4) the small-scale anthropic transport of RGFO PKA nodules. While expanding the archaeological knowledge on RGFO PKA natural availability and their technological exploitation in the Pali Aike Volcanic Field, data provided here agree with the argument that the Gallegos-Chico interfluve was a key provisioning area for this lithic raw material as previous works showed.

Key words: secondary lithic sources, nodules, land use, Patagonia

\section{Introducción}

El estudio de las fuentes de materias primas líticas y su explotación por parte de cazadores-recolectores es un tema recurrente en la arqueología de Argentina (Alberti 2012; Apolinaire 2018; Bobillo y Hocsman 2015; Borrazzo et al. 2015; Catella et al. 2017; Cortegoso et al. 2020; entre muchos otros). En Patagonia sur continental, y a lo largo de los años, se desarrollaron diversas aproximaciones focalizadas en conocer la disponibilidad y distribución de las rocas aptas para la talla con el objetivo de evaluar la movilidad, interacción y estrategias tecnológicas implementadas por las poblaciones en el pasado (Belardi et al. 2015; Charlin 2009; Franco 2002; Skarbun 2011; Stern 2018, entre otros).

En el caso específico del campo volcánico Pali Aike (CVPA), sector argentino, se han realizado variados estudios sobre la estructura de los recursos líticos y su potencial para la talla (Charlin 2009; Charlin y Pallo 2015; Nami 1994; entre otros). Nuestro trabajo aborda el estudio arqueológico de la disponibilidad y uso de las materias primas conocidas como Rocas de Grano Fino Oscuras (RGFO) tipo Potrok Aike (PKA) en el sector central del interfluvio de los ríos Gallegos y Chico (IGCh). Se trata de rocas que fueron sistemáticamente seleccionadas para la elaboración de diversos tipos artefactuales en el CVPA y exhiben una distribución arqueológica a escala regional (Balirán 2021; Charlin y Pallo 2013; Charlin et al. 2011; entre otros).

En ese marco, este trabajo tiene como objetivos 1) informar la localización de las fuentes potenciales de aprovisionamiento de la variedad PKA, 2) evaluar la abundancia, distribución y características de los nódulos allí recuperados, y 3) explorar las variaciones distribucionales existentes en su explotación en un segmento del IGCh para el que no se contaba con datos sistemáticos. Dado que la obtención de este recurso lítico pudo ser integrada al ciclo más amplio de actividades desarrolladas por cazadores-recolectores (Binford 1979; Charlin y Pallo 2015), se propone que la adquisición de esta materia prima habría sido de manera directa. De esta forma de obtención, considerando a su vez estrategias de economía de materia prima (Odell 1996), se derivan expectativas arqueológicas específicas tales como el decrecimiento en la frecuencia artefactual, la reducción del tamaño de los artefactos y la disminución de la reserva de corteza en las piezas a medida que aumenta la distancia a la fuente de abastecimiento (Renfrew 1977; entre otros). 


\section{Antecedentes}

El CVPA cubre un área de ca. 3.000 - $4.500 \mathrm{~km}^{2}$ en el extremo sur de Patagonia continental (D' Orazio et al. 2000; Skewes 1978). El espacio abordado en este trabajo se encuentra en el centro del campo volcánico, sector argentino, delimitado por los ríos Gallegos y Chico (Borrazzo et al. 2019). Corresponde a un ambiente de estepa seca con lagunas temporales y escasas precipitaciones anuales (200 a $300 \mathrm{~mm}$ ) (Oliva et al. 2001). Este sector del interfluvio registra pocos reparos rocosos y baja productividad primaria en oposición al sector sur del CVPA, donde los abrigos son más frecuentes y la productividad primaria es mayor (Barberena 2008).

El CVPA posee ocupaciones humanas comprendidas entre $c a .11 .000$ y 100 años AP (Bird 1988; Nami 1999; entre otros). Las características del registro arqueológico lítico -puntas de proyectil asignadas a los períodos IV y V de Bird (1988)- y los fechados radiocarbónicos del área central del IGCh (ca. 2700-300 años AP) sitúan a las ocupaciones cazadoras-recolectoras durante el Holoceno tardío (Borrazzo et al. 2019; L'Heureux et al. 2020, 2021).

Las investigaciones previas de la base regional de recursos líticos en el CVPA exploraron marginalmente el centro del IGCh, objeto de este trabajo (Charlin 2009; Charlin y Pallo 2015; Nami 1994, 1999, 2009). De modo general, esos estudios mostraron que los grupos humanos utilizaron las materias primas líticas disponibles en fuentes secundarias -depósitos glacifluviales- (Charlin 2005; ver también Gómez Otero 1986-87; Nami 1994, 1999). Asimismo, destacaron la función de algunas lagunas (Potrok Aike, Pali Aike y lagunas de cerro Mackenzie), cauces del río Chico y espacios erosionados como fuentes potenciales de basalto (Gómez Otero 1986-87), rocas volcánicas negras (RVN sensu Nami 2000: 241) y/o rocas silíceas, entre otras materias primas (Gómez Otero 1986-87; Nami 1994, 1999, 2009). Por su parte, las investigaciones de Charlin mostraron que los depósitos glacifluviales están dominados por dacitas de colores claros y Rocas de Grano Fino Oscuras (RGFO) de distintos tipos petrográficos, registrándose en menor proporción otras materias primas como jaspe o calcedonia (Charlin 2009; Charlin y Pallo 2015).

Análisis geoquímicos y petrográficos permitieron identificar dentro del grupo de las RGFO una variedad de origen volcánico (Charlin y D'Orazio 2010) denominada "Potrok Aike" (Charlin y Pallo 2013: 309). Se trata de una dacita vítrea que puede ser identificada macroscópicamente por presentar corteza vesicular, pasta de color negro oscuro y brilloso, fenocristales de tamaños pequeños y en baja proporción ( 10-5 \% del volumen de la roca) y muy buena calidad para la talla (Charlin y D'Orazio 2010; Charlin y Pallo 2013). Los estudios realizados mostraron que su disponibilidad natural es relativamente abundante en algunas lagunas del IGCh ubicadas al este y al oeste del sector abordado en este trabajo (Potrok Aike1, Seca INTA, Carolina 1, Carolina 2 y Bismarck). Por ello, se señaló a las lagunas del IGCh como fuentes potenciales de abastecimiento de RGFO PKA y se propuso una disminución en la disponibilidad de la 
variedad PKA de oeste a este (Charlin y Pallo 2013). Es importante destacar que esta materia prima fue también reconocida en baja frecuencia en el curso medio e inferior del río Gallegos (Eas. Las Buitreras, Primero de Mayo y Palermo Aike) y en sectores más alejados como río El Zurdo -al oeste del CVPA- y Punta Loyola -costa Atlántica(Charlin y D'Orazio 2010; Charlin y Pallo 2013).

Dentro del grupo de las RGFO, la variedad PKA fue la más utilizada para la confección de artefactos en el CVPA² (Charlin y Pallo 2013). Aunque la disponibilidad natural de la RGFO PKA se encuentra mayormente limitada al IGCh, esta materia prima registra una distribución espacial bajo la forma de artefactos que excede los límites del CVPA y alcanza espacios más alejados como La Verdadera Argentina Noroeste- (Balirán 2021), Laguna Cóndor y río Penitente -Oeste- (Belardi et al. 2020; Charlin et al. 2011), Cañadón GAP -Este- (Charlin et al. 2010) y estrecho de Magallanes Sur- (Charlin y D'Orazio 2015). En ese sentido, se ha planteado que los artefactos elaborados con RGFO PKA constituyen una de las líneas de evidencia que apoyan la existencia de un núcleo poblacional en el CVPA con un rango de acción particular durante el Holoceno tardío (Charlin et al. 2011; entre otros). A su vez, esta variedad de roca ha sido considerada como un bien vinculado a la interacción con otros núcleos poblacionales de la región (Belardi et al. 2020; entre otros).

Los trabajos de investigación realizados en el marco del PICT 2014-2061 permitieron ampliar la cobertura arqueológica del sector central del IGCh (Borrazzo et al. 2019; L'Heureux et al. 2020). Los estudios tecnológicos mostraron que la RGFO PKA fue la materia prima más utilizada para confeccionar artefactos en el sector central del IGCh (Borrazzo et al. 2019), tal como había sido observado en espacios vecinos (Charlin y D’Orazio 2010; Charlin y Pallo 2013). Asimismo, se identificó el empleo de la técnica bipolar en la explotación de rodados de RGFO PKA y la representación local de todos los estadios de talla sobre esta materia prima -incluyendo la talla bifacial- (Borrazzo y Cirigliano 2020; Borrazzo et al. 2019; Cirigliano et al. 2020). Con respecto al uso de los espacios por parte de cazadores-recolectores en el IGCh central, se reconoció la ocupación intensa ${ }^{3}$ y recurrente (planificada) de un reparo rocoso (Alero Clark) y de algunos sectores lagunares (Ea. Otern Aike y Eas. La Argentina-Tres Lagunas) con disponibilidad de RGFO PKA y una menor distancia a los cursos fluviales (Borrazzo y Cirigliano 2020). En esos espacios, considerados nodos de asentamiento, se realizaron actividades múltiples como la obtención, procesamiento y consumo de presas, y el aprovisionamiento de rocas y la manufactura de artefactos líticos (Borrazzo y Cirigliano 2020; Borrazzo et al. 2019). Se registraron instrumentos multipropósitos de grandes dimensiones (e.g. yunque, molino, percutor) y/o ecofactos (nódulos de calcedonia volcánica y RGFO PKA) que podrían indicar equipamiento de esos mismos espacios (sensu Binford 1979; v. Borrazzo y Cirigliano 2020).

En este trabajo se presentan los relevamientos sistemáticos de las fuentes secundarias de RGFO PKA localizadas en el sector central del IGCh (Figura 1), se caracterizan sus nódulos y se analizan los artefactos confeccionados con esta materia 
prima procedentes del mismo sector. Luego, se integra la base regional de RGFO PKA construida para el área de estudio con la información tecnomorfológica obtenida del análisis de los conjuntos artefactuales. Se considera que la identificación de criterios técnicos priorizados en la explotación de esta materia prima contribuye a la caracterización de modos de uso del espacio particulares dentro del CVPA, así como también a entender el comportamiento tecnológico de los cazadores-recolectores de Patagonia meridional. Para completar el objetivo, los resultados obtenidos son discutidos en el marco de la organización tecnológica (Nelson 1991) e integrados en el contexto general del CVPA y áreas aledañas (Belardi et al. 2020; Borrazzo y Cirigliano 2020; Charlin 2009; Charlin y Pallo 2013; Nami 1999, 2009).

\section{Metodología}

Los muestreos sistemáticos de rocas y de artefactos líticos se efectuaron en el marco de una meso-transecta que cubrió una longitud de ca. $30 \mathrm{~km}$ (Borrazzo y Cirigliano 2020) en una porción del IGCh localizada a los $\mathrm{O} 70^{\circ}$. De norte a sur, los espacios investigados se encuentran en las Eas. $1^{\circ}$ de Mayo, Otern Aike, Don Raúl, La Argentina, Tres Lagunas, Osasuna y Pali Aike. El extremo norte de la meso-transecta se localizó próximo al río Gallegos, mientras que el extremo sur se ubicó inmediatamente al norte del río Chico (Figura 1).

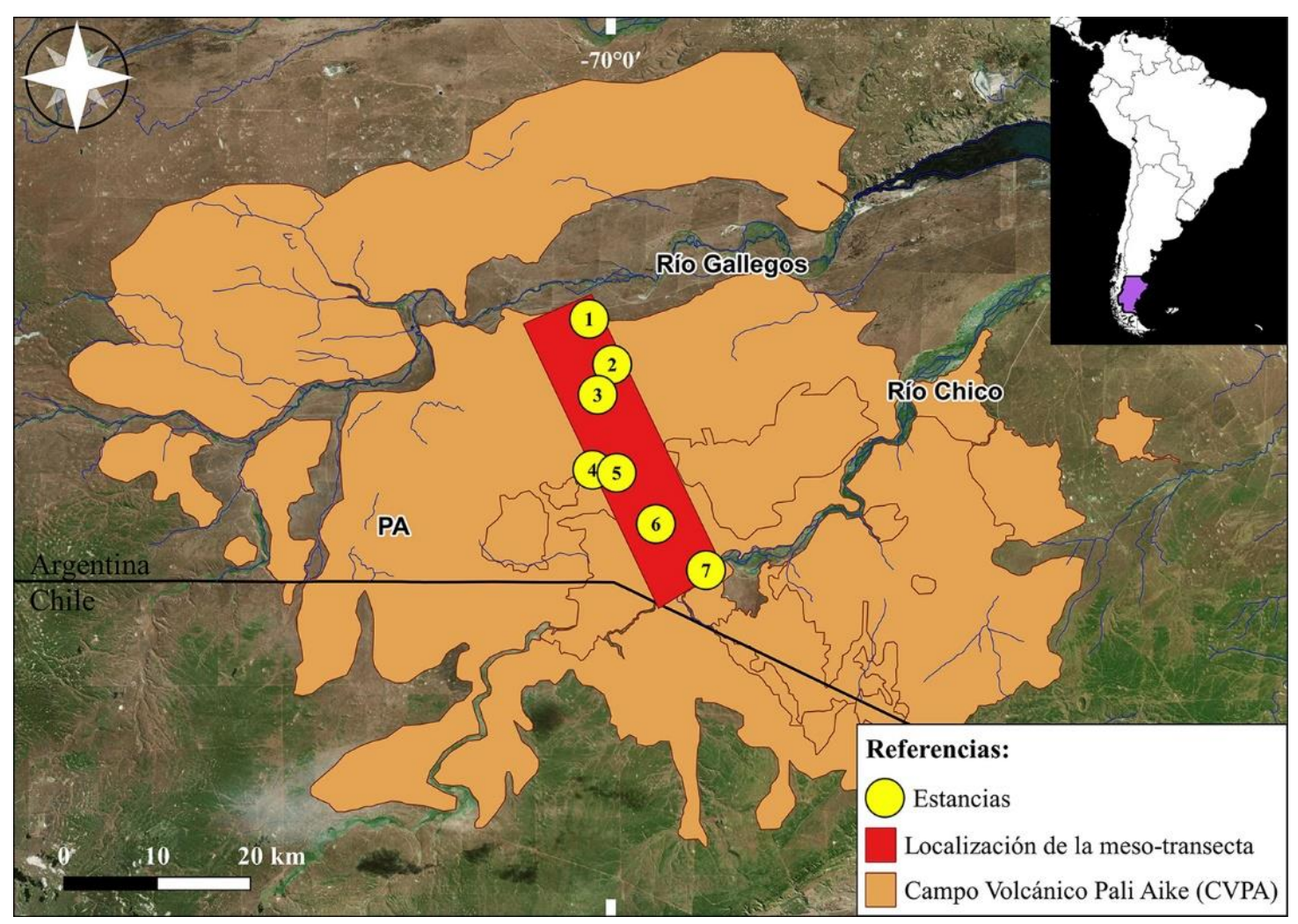

Figura 1. Área de estudio de este trabajo. Localización de la meso-transecta y estancias. Referencias: $1.1^{\circ}$ de Mayo; 2. Otern Aike; 3. Don Raúl; 4. La Argentina; 5. Tres Lagunas; 6. Osasuna; 7. Pali Aike; PA. Laguna Potrok Aike. 
Las tareas de campo buscaron evaluar la disponibilidad natural de las RGFO PKA en fuentes secundarias (sensu Nami 1992) para conocer su distribución espacial. Los muestreos sistemáticos de materias primas tuvieron por objetivo recolectar y testear rocas de calidades aptas para la talla y fueron efectuados por una persona, durante cinco minutos, siguiendo la propuesta de Franco y Borrero (1999). Se registró la frecuencia de rocas y el rendimiento de nódulos de RGFO PKA. En este trabajo se propuso al rendimiento como el total de nódulos de RGFO PKA recuperados por una persona en cinco minutos y se lo consignó como muy alto ( $\geq 15$ nódulos), alto (14-10 nódulos), intermedio (9-5 nódulos) y bajo (1-4 nódulos). En el caso de no recuperar RGFO PKA en los muestreos, se extendió el tiempo de búsqueda (e.g. Bajo 1, Bajo 2 y Laguna Pali Aike). En este trabajo se presenta la información propia generada para las RGFO PKA y se retoman por su pertinencia los datos publicados de muestreos realizados en la Ea. $1^{\circ}$ de Mayo (Charlin y Pallo 2015) y en proximidades del río Chico (L'Heureux et al. 2020).

La identificación de las materias primas se realizó macroscópicamente según colección de referencia de rocas alojadas en el IMHICIHU-CONICET (Charlin 2009; Charlin y D'Orazio 2010, 2015; Charlin et al. 2010). Se consignó la calidad para la talla (sensu Aragón y Franco 1997) en mala, regular, buena, muy buena y excelente y la morfología de los nódulos en compacto, elongado/ prolado y muy aplanado (Graham y Midgley 2000, 2003; Sneed y Folk 1958). Asimismo, se señaló la longitud del eje mayor de los nódulos $(\mathrm{cm})$ y su volumen $\left(\mathrm{cm}^{3}\right)$.

Los conjuntos artefactuales analizados proceden de contextos de superficie. Para su recolección se utilizaron cuadrículas y transectas lineales. Dados los objetivos de este trabajo, aquí abordamos exclusivamente aquellos artefactos $(\mathrm{N}=2154)$ elaborados con RGFO PKA (los resultados globales pueden consultarse en Borrazzo y Cirigliano 2020).

Para el análisis tecnológico de los artefactos líticos se siguieron los lineamientos metodológicos propuestos por Aschero $(1975,1983)$. En líneas generales, se consideró tipo y calidad de la materia prima, clase de artefacto (núcleo, desecho de talla e instrumento), porcentaje de reserva de corteza en cinco intervalos (0 \%, 1-25 \%, 26-50 \%, 51-75 \% y 76$100 \%)$ y tamaño a partir de la grilla elaborada por Franco (2002). Los artefactos fueron agrupados según su tamaño de grilla en muy pequeños $(0,1-20 \mathrm{~mm})$, pequeños $(20,1-40$ $\mathrm{mm})$, mediano-pequeños (40,1 - $60 \mathrm{~mm})$, mediano-grandes $(60,1-80 \mathrm{~mm})$, grandes $(80,1$ $100 \mathrm{~mm}$ ) y muy grandes (> 100,1 mm). Estas variables fueron consideradas sobre la base de las expectativas asociadas con el acceso directo a las materias primas y su transporte bajo la forma de artefactos a otros espacios donde ésta no se encuentra naturalmente disponible. Adicionalmente, se consideró el supuesto de que en nódulos cuya superficie natural se encuentra completamente cubierta por corteza (como es el caso de los rodados explotados en la región de estudio), el tamaño y la reserva de corteza disminuyen conforme avanza la reducción (Clarkson 2008; Ericson 1984; Morello 2005; Phillipps y Holdaway 2016; Renfrew 1977; Shiner 2004; entre otros). A los fines de lograr una primera 
aproximación y obtener tendencias promediadas acerca de la abundancia y explotación de la RGFO PKA, se analizaron todos los artefactos recuperados sin discriminar su estado (entero o fragmentado). Se compararon las frecuencias observadas en los distintos sectores mediante el test de $\mathrm{Chi}^{2}$ (Past, Hammer et al. 2001), utilizando la estimación de la probabilidad asociada de Monte Carlo por la existencia de casos con frecuencias muy bajas o nulas.

La localización de los muestreos de rocas y las características de distintas variables de los nódulos de RGFO PKA fueron volcadas en una base de datos del Software QGIS 3.10 para graficar densidad y distribución espacial de tamaños y formas de los nódulos. Este mismo procedimiento se realizó con los artefactos de RGFO PKA con el objetivo de explorar la abundancia e intensidad de explotación de esta materia prima en los sectores muestreados.

\section{Resultados}

\section{Las fuentes de RGFO PKA}

Los muestreos sistemáticos de materias primas $(\mathrm{N}=14)$ se ejecutaron en las márgenes o fondos de bajos lagunares, en sectores erosionados inmediatamente próximos a éstos y en manantiales. Se seleccionaron mayormente espacios que presentaban disponibilidad de nódulos para su testeo, aunque también se escogieron en menor medida sectores que a simple vista no presentaban rocas de aptitud para la talla.

Se recolectó un total de 246 nódulos en los muestreos. Entre los ejemplares de calidades aptas para la talla se reconocieron rocas volcánicas ácidas y mesosilícicas de colores claros, distintas RGFO volcánicas (incluida la variedad PKA), rocas silicificadas de colores claros de distinto origen, jaspe, madera petrificada y calcedonia.

La RGFO PKA constituye el 43,09 \% del total de las rocas recuperadas en los muestreos (Tabla 1 y Figura 2). Los 106 nódulos de RGFO PKA se encuentran completamente cubiertos con corteza vesicular y corresponden en su mayoría a rocas de muy buena calidad para la talla $(92,46 \%)$ (Figura 3), aunque también se reconocen algunos ejemplares de aptitud buena $(6,60 \%)$ y mala $(0,94 \%)$. A continuación, se presentan los resultados sobre la disponibilidad de RGFO PKA y se adiciona la información provista por muestreos asistemáticos y recolecciones selectivas.

Como se observa en la Tabla 1, la abundancia de RGFO PKA no es homogénea en la meso-transecta (Figura 4). El promedio de los muestreos realizados en cada uno de los sectores permite observar diferencias en la cantidad de nódulos recuperados. Laguna La Argentina presentó un rendimiento muy alto, mientras que otros sectores registraron rendimientos altos (Laguna Otern Aike 1), intermedios (Laguna Otern Aike 2) y bajos (Manantial, Laguna Tres Lagunas). Finalmente, los hallazgos fueron nulos en Bajo 1, Bajo 2 y Laguna Pali Aike. 


\begin{tabular}{|c|c|c|c|c|c|c|c|c|}
\hline \multirow{2}{*}{ Estancia } & \multirow{2}{*}{ Sector muestreado } & \multirow{2}{*}{$\begin{array}{l}\text { Formación Geológica } \\
\text { (Panza y Sacomani 2015) }\end{array}$} & \multicolumn{3}{|c|}{ Nódulos } & \multirow[b]{2}{*}{ Rend. } & \multirow{2}{*}{$\begin{array}{l}\text { Vol. } \\
\text { máximo } \\
\left(\mathrm{cm}^{3}\right)\end{array}$} & \multirow{2}{*}{$\begin{array}{l}\text { Rec. } \\
\text { Art. }\end{array}$} \\
\hline & & & $\mathbf{N}$ & \multicolumn{2}{|c|}{$\begin{array}{l}\text { RGFO PKA } \\
\mathbf{N} \quad \%\end{array}$} & & & \\
\hline \multirow{2}{*}{$\begin{array}{l}1^{\circ} \mathrm{de} \\
\text { Mayo }\end{array}$} & Bajo 1 & $\begin{array}{l}10^{*} \text {. Dep. fluviales aterrazados } \\
\text { del río Gallegos (Niveles I y II) }\end{array}$ & $10^{4}$ & 0 & 0 & - & - & Si \\
\hline & Manantial & $\begin{array}{l}\text { 1a. Formación Santa Cruz } \\
\text { parcialmente cubierta }\end{array}$ & 12 & 4 & 3,77 & Bajo & 166,71 & $\mathrm{No}^{5}$ \\
\hline \multirow{5}{*}{$\begin{array}{l}\text { Otem } \\
\text { Aike }\end{array}$} & $\begin{array}{l}\text { Laguna Otern Aike 1, } 1 \\
\text { (margen sur) }\end{array}$ & $\begin{array}{l}\text { 42. Dep. aluviales y coluviales } \\
\text { indiferenciados; } 37 \text {. Sed. finos } \\
\text { de bajos y lagunas }\end{array}$ & 19 & 10 & 9,43 & Alto & \multirow[t]{2}{*}{827,58} & \multirow{2}{*}{ Si } \\
\hline & $\begin{array}{c}\text { Laguna Otern Aike 1,2 } \\
\text { (margen este) }\end{array}$ & $\begin{array}{l}\text { 42. Dep. aluviales y coluviales } \\
\text { indiferenciados; } 37 . \text { Sed. finos } \\
\text { de bajos y lagunas }\end{array}$ & 30 & 19 & 17,92 & $\begin{array}{l}\text { Muy } \\
\text { alto }\end{array}$ & & \\
\hline & $\begin{array}{l}\text { Laguna Otern Aike 2,1 } \\
\text { (sector oeste, cárcava en } \\
\text { lomada, punto elevado) }\end{array}$ & $\begin{array}{l}\text { 42. Depósitos aluviales y } \\
\text { coluviales indiferenciados }\end{array}$ & 35 & 9 & 8,49 & $\begin{array}{c}\text { Interm } \\
\text { edio }\end{array}$ & \multirow{3}{*}{434,08} & \multirow{3}{*}{ Sí } \\
\hline & $\begin{array}{l}\text { Laguna Otern Aike 2, } 2 \\
\text { (sector oeste, cárcava en } \\
\text { lomada, punto inter.) }\end{array}$ & $\begin{array}{l}\text { 42. Dep. aluviales y coluviales } \\
\text { indiferenciados }\end{array}$ & 22 & 10 & 9,43 & Alto & & \\
\hline & $\begin{array}{c}\text { Laguna Otern Aike 2,3 } \\
\text { (fondo y margen de } \\
\text { laguna) }\end{array}$ & $\begin{array}{l}\text { 42. Dep. aluviales y coluviales } \\
\text { indiferenciados; } 37 \text {. Sed. finos } \\
\text { de bajos y lagunas }\end{array}$ & 21 & 8 & 7,55 & $\begin{array}{l}\text { Interm } \\
\text { edio }\end{array}$ & & \\
\hline Don Raúl & Bajo 2 & 17a. Basalto Pali Aike & 2 & 0 & 0 & - & - & Si \\
\hline \multirow[b]{2}{*}{$\begin{array}{c}\mathrm{La} \\
\text { Argentina }\end{array}$} & $\begin{array}{l}\text { Laguna La Argentina, } 1 \\
\text { (sector norte) }\end{array}$ & $\begin{array}{l}\text { 30. Dep. de antiguas playas y } \\
\text { cordones litorales lacustres }\end{array}$ & 20 & 19 & 17,92 & $\begin{array}{l}\text { Muy } \\
\text { alto }\end{array}$ & \multirow[b]{2}{*}{1090,73} & \multirow[b]{2}{*}{ Si } \\
\hline & $\begin{array}{l}\text { Laguna La Argentina, } 2 \\
\text { (sector norte) }\end{array}$ & $\begin{array}{l}\text { 30. Dep. de antiguas playas y } \\
\text { cordones litorales lacustres } \\
\text { 37. Sed. finos de bajos y lagunas }\end{array}$ & 27 & 23 & 21,71 & $\begin{array}{l}\text { Muy } \\
\text { alto }\end{array}$ & & \\
\hline \multirow{2}{*}{$\begin{array}{c}\text { Tres } \\
\text { Laguna }\end{array}$} & $\begin{array}{c}\text { Laguna Tres Lagunas, } 1 \\
\text { (sector sur) }\end{array}$ & $\begin{array}{l}\text { 30. Dep. de antiguas playas y } \\
\text { cordones litorales lacustres } \\
\text { 37. Sed. finos de bajos y lagunas }\end{array}$ & 8 & 2 & 1,89 & Bajo & \multirow{2}{*}{1351,91} & \multirow{2}{*}{ Si } \\
\hline & $\begin{array}{l}\text { Laguna Tres Lagunas, } 2 \\
\text { (sector sur) }\end{array}$ & $\begin{array}{l}\text { 30. Dep. de antiguas playas y } \\
\text { cordones litorales lacustres } \\
\text { 37. Sed. finos de bajos y lagunas }\end{array}$ & 6 & 2 & 1,89 & Bajo & & \\
\hline \multirow{2}{*}{ Osasuna } & $\begin{array}{l}\text { Laguna Pali Aike, } 1 \\
\text { (fondo de laguna) }\end{array}$ & 37. Sed. finos de bajos y lagunas & 18 & 0 & 0 & - & \multirow[b]{2}{*}{ - } & \multirow{2}{*}{ Si } \\
\hline & $\begin{array}{l}\text { Laguna Pali Aike, } 2 \\
\text { (fondo de laguna) }\end{array}$ & 37. Sed. finos de bajos y lagunas & 16 & 0 & 0 & - & & \\
\hline Total & - & - & 246 & 106 & 100 & - & - & - \\
\hline
\end{tabular}

Tabla 1. Muestreos sistemáticos de materias primas. Referencias: Rend. (Rendimiento), Vol. Máx. (Volumen máximo de nódulo), Rec. Art. (Recolección de Artefactos), Dep. (Depósitos), Sed. (Sedimentos), * los números se corresponden con la simbología cartográfica de la Hoja Geológica.

Es importante señalar que las recolecciones selectivas de RGFO PKA realizadas en Ea. Don Raúl y los relevamientos en los que únicamente se buscó reconocer y recuperar RGFO PKA sobre la margen norte del río Chico y márgenes de la Laguna Pali Aike permitieron complementar la información sobre la disponibilidad de este recurso. Se identificaron tres nódulos de RGFO PKA en Ea. Don Raúl fuera del tiempo de muestreo y entre 100 y 1000 m de distancia del Bajo 2. Las márgenes de Laguna Pali Aike y los espacios adyacentes a la margen norte del río Chico no entregaron nódulos de RGFO PKA (L'Heureux et al. 2020). 


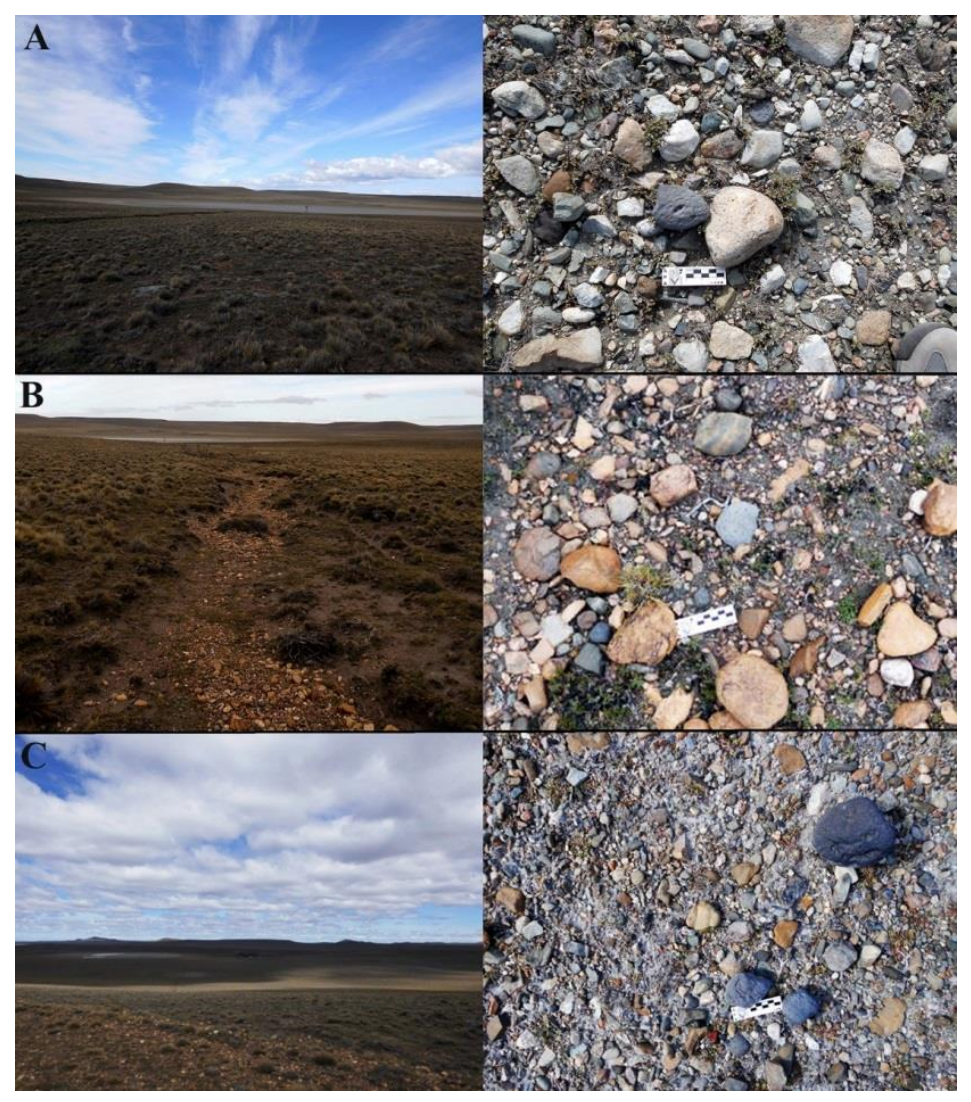

Figura 2. Fuentes secundarias muestreadas con disponibilidad de RGFO PKA (descriptas en Tabla 1) . Referencias: A. Laguna Otern Aike 2, 3; B. Laguna Otern Aike 2, 2; C. Laguna La Argentina, 2.

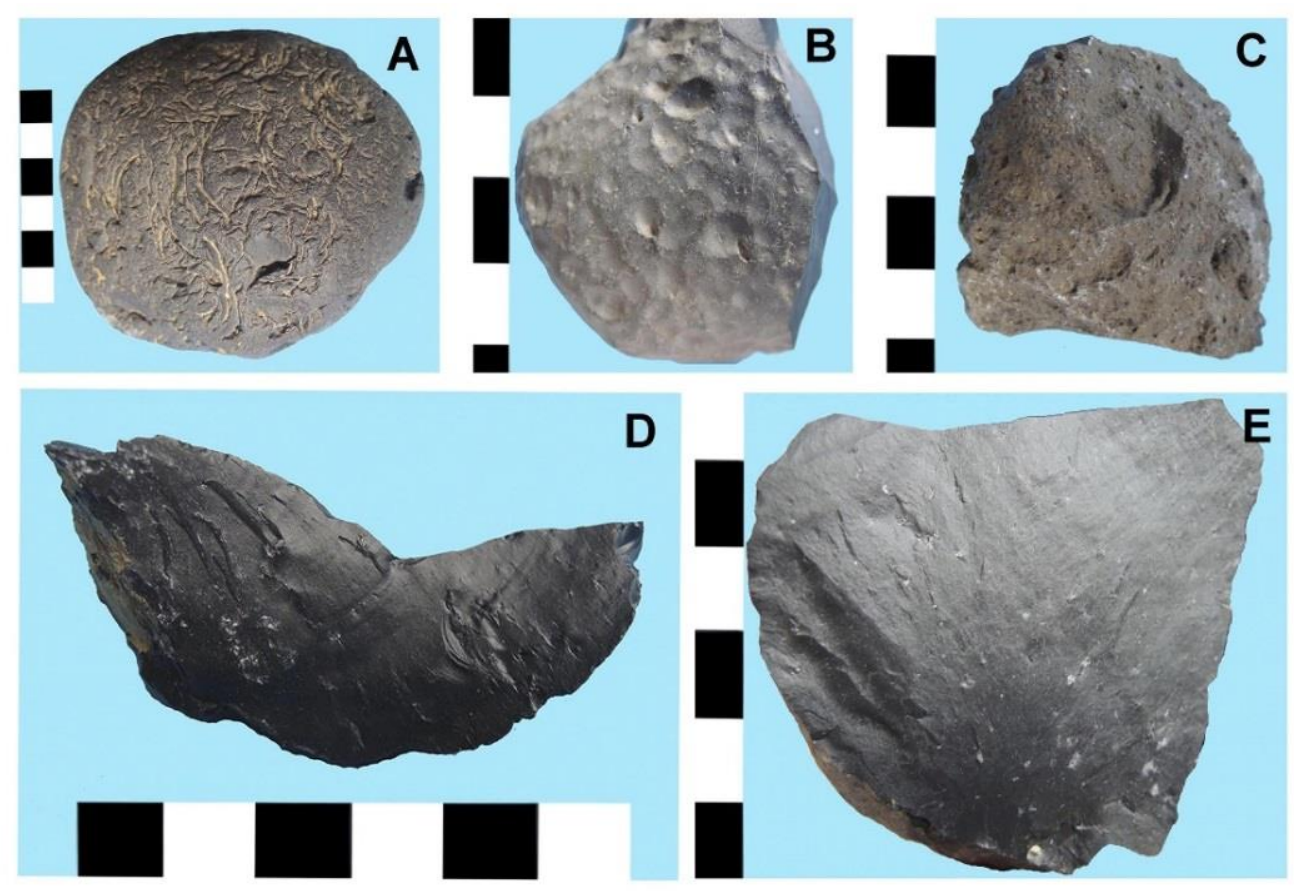

Figura 3. Roca de grano fino oscura tipo Potrok Aike. Referencias: A, B y C. Detalle de corteza; D y E. Detalle de fractura fresca. 


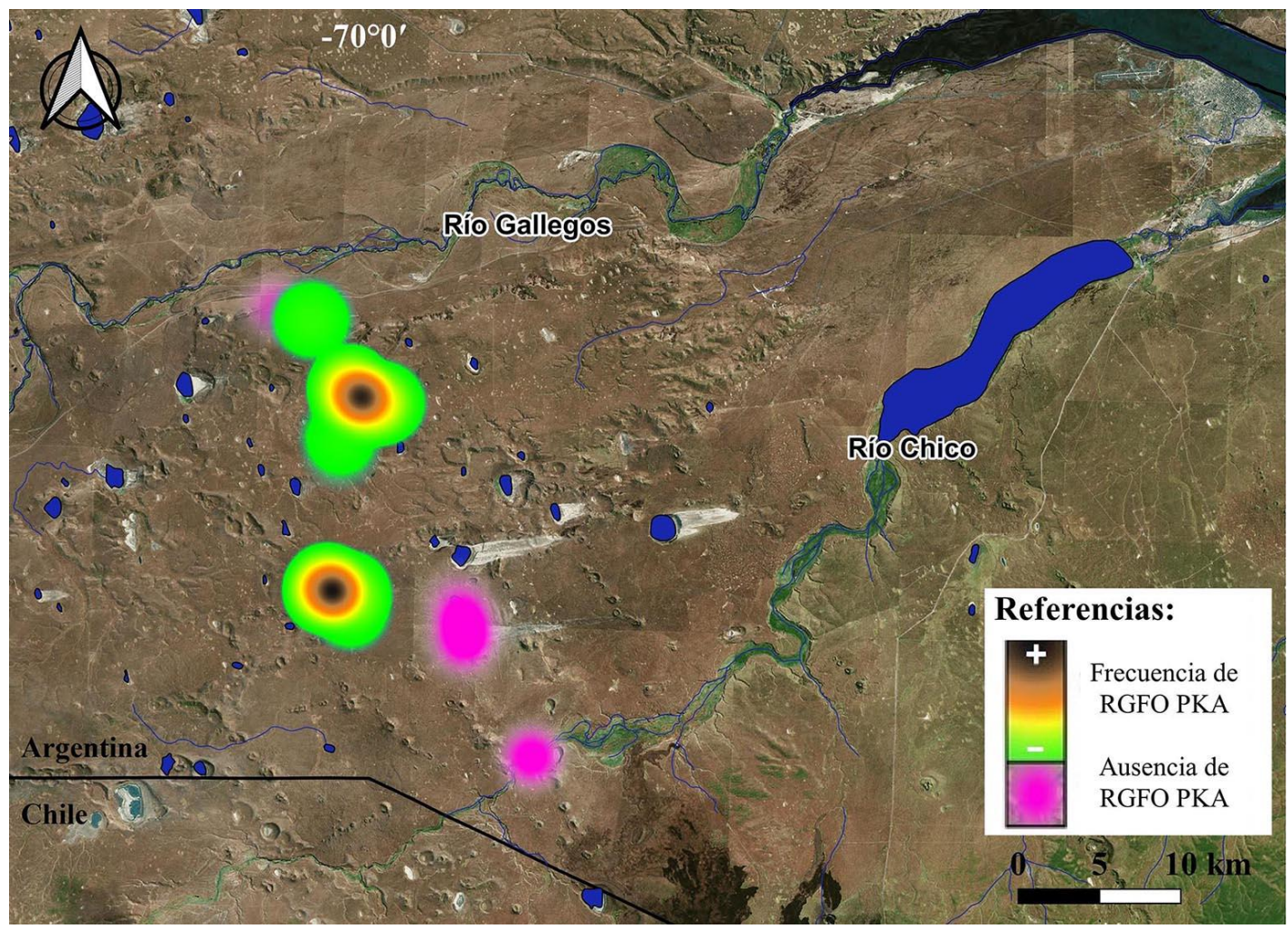

Figura 4. Disponibilidad de RGFO PKA en el sector central del IGCh. Se incluye la información de los muestreos sistemáticos, relevamientos intensos y recolecciones selectivas. Se incorporan al mapa de calor tres muestreos sistemáticos realizados en Ea. $1^{\circ}$ de Mayo y donde sólo se recuperó un nódulo de RGFO PKA fuera del tiempo de muestreo (Charlin y Pallo 2013).

En vista de los datos obtenidos, la mayor abundancia de RGFO PKA se localiza en la porción central de la meso-transecta, con un muy alto rendimiento en Laguna La Argentina. Es significativo señalar que se detectaron variaciones en la disponibilidad de este recurso a pequeña escala. Por ejemplo, este es el caso de Laguna Tres Lagunas en donde se registró bajo rendimiento de RGFO PKA en los muestreos de materia primas realizados en su margen sur. No obstante, en un sector de deflación de la margen Noreste, las prospecciones superficiales en el marco de una transecta de reconocimiento del área permitieron detectar más nódulos de RGFO PKA ( $n=12)$. Por otra parte, los espacios localizados en los extremos de la meso-transecta, es decir próximos a los ríos Gallegos y Chico, registran bajo rendimiento o ausencia de nódulos de RGFO PKA respectivamente.

Con respecto al eje mayor y volumen de los nódulos, al agrupar los muestreos por sector se observa una tendencia creciente de ambas variables de norte a sur. En ese sentido, los pocos ejemplares recuperados en el muestreo del Manantial (Ea. $1^{\circ}$ de Mayo) registran los valores más bajos para las longitudes mínimas $(2,65 \mathrm{~cm})$ y máximas $(7,60 \mathrm{~cm})$, mientras que el conjunto de Laguna Tres Lagunas muestra los valores más elevados para las longitudes mínimas $(9,70 \mathrm{~cm})$ y máximas $(15,50 \mathrm{~cm})$ (Figura 5a). Laguna La Argentina y Laguna Otern Aike 1 y 2 exhiben gran variabilidad de tamaños, lo que podría estar vinculado con la abundancia de los nódulos testeados. Las dimensiones promedio para el eje 
mayor de los nódulos son de norte a sur las siguientes: 5,07 cm (Manantial), 7,73 cm (Laguna Otern Aike 1), 6,28 cm (Laguna Otern Aike 2), 7,55 cm (Laguna La Argentina) y 13,05 cm (Laguna Tres Lagunas). En líneas generales, los muestreos están dominados por nódulos con longitudes mediano-pequeñas ( $>5-\leq 10 \mathrm{~cm} ; 65,09 \%)$ y pequeñas $(>0-\leq 5 \mathrm{~cm} ; 18,86 \%)$; aquéllos de dimensiones mediano-grandes (> $10-\leq 15 \mathrm{~cm} ; 15,09 \%)$ y grandes $(>15$ $\mathrm{cm} ; 0,94 \%)$ son menos frecuentes.

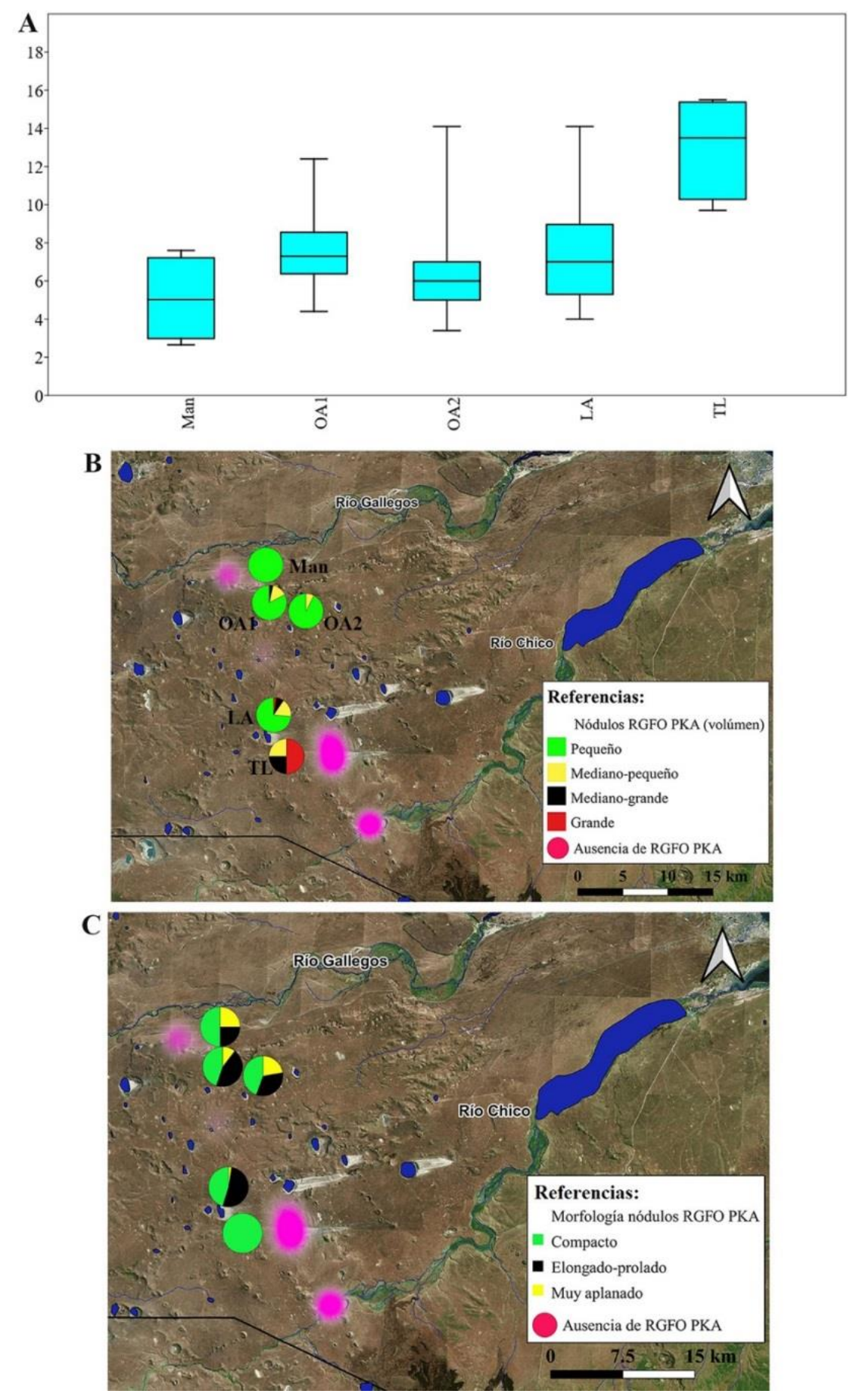

Figura 5. Características de los nódulos de RGFO PKA por sector muestreado. A) Eje mayor $(\mathrm{cm})$, B) Volumen $\left(\mathrm{cm}^{3}\right)$, y C) Morfología. Referencias: Man= Manantial; OA1= Laguna Otern Aike 1; OA2= Laguna Otern Aike 2; LA= Laguna La Argentina; TL= Laguna Tres Lagunas. 
En cuanto al volumen, hay una predominancia de nódulos de tamaños pequeños ( $\leq 300 \mathrm{~cm}^{3} ; 79,24 \%$ ) (Figura 5b). En el extremo norte de la meso-transecta, sector del Manantial, éstos constituyen el 100 \% de la muestra. Aquí se registran los volúmenes mínimos $\left(10,72 \mathrm{~cm}^{3}\right)$ y máximos $\left(166,71 \mathrm{~cm}^{3}\right)$ más pequeños de los muestreos sistemáticos realizados en la meso-transecta. Los tamaños pequeños también son dominantes en Laguna Otern Aike 1 (82,76 \%), Laguna Otern Aike 2 (92,59 \%) y Laguna La Argentina (73,81 \%). Los guijarros de tamaño mediano-pequeños (> $300-\leq$ $650 \mathrm{~cm}^{3}$ ) son poco frecuentes y se reconocen en baja proporción en Laguna Otern Aike 1 (13,79 \%), Laguna Otern Aike 2 (7,41 \%), Laguna La Argentina (16,67 \%) y Laguna Tres Lagunas (25\%). Los nódulos de volumen mediano-grande $\left(>650-\leq 1000 \mathrm{~cm}^{3}\right)$ son escasos y se registraron en Laguna Otern Aike 1 (3,45 \%), Laguna La Argentina $(7,14 \%)$ y Laguna Tres Lagunas (25\%). Por último, sólo se reconocieron tres guijarros de tamaño grande $\left(>1000 \mathrm{~cm}^{3}\right)$ en Laguna La Argentina $\left(\mathrm{n}=1 ; 2,38 \% ; 1090,73 \mathrm{~cm}^{3}\right)$ y Laguna Tres Lagunas (n=2; $50 \% ; 1250,44$ y 1351,91 $\left.\mathrm{cm}^{3}\right)$. Cabe destacar que el ejemplar grande de Laguna La Argentina es de mala calidad para la talla debido a la presencia de planos de oxidación. Aquí también es significativo señalar que los nódulos recuperados fuera del tiempo de muestreo en Ea. Don Raúl son de tamaño pequeño, siendo su eje mayor $\leq 5,90 \mathrm{~cm}$. Asimismo, los nódulos identificados en la margen Noreste de Laguna Tres Lagunas presentan variabilidad de tamaños (pequeños a grandes), con ejes mayores comprendidos entre 5,20 y 15,20 cm.

Se detectaron nódulos con morfologías compactas (47,17\%), elongadas-proladas $(42,45 \%)$ y muy aplanadas-elongadas (10,38 \%) (Figura 5c). Los guijarros compactos, sin plataformas naturales adecuadas para la talla ( $v$. Shelley 1993), se encuentran en todos los sectores y en altas frecuencias: Laguna Tres Lagunas (100\%), Manantial $(50,00 \%)$, Laguna La Argentina (45,24 \%) y Laguna Otern Aike 1 (44,83\%) y 2 $(44,44 \%)$. Por otra parte, los nódulos elongados-prolados, con plataformas naturales apropiadas para la talla, se encuentran en orden de importancia en Laguna La Argentina (52,38 \%), Laguna Otern Aike 1 (44,83\%) y 2 (33,33\%), y Manantial $(25,00 \%)$.

En líneas generales, los mayores rendimientos de RGFO PKA en el área de la meso-transecta se registraron en Laguna La Argentina y Laguna Otern Aike 1. Los nódulos de mayor volumen se reconocieron únicamente en baja frecuencia en Laguna La Argentina y Laguna Tres Lagunas. Dado que el nódulo de tamaño grande recuperado en Laguna La Argentina es de mala calidad para la talla, únicamente Laguna Tres Lagunas entregó nódulos de volúmenes elevados y aptos para la talla, aunque aquí el rendimiento de la RGFO PKA es relativamente menor que en otros espacios. Por último, la morfología más apropiada para la talla (elongado-prolado) se identificó en Laguna La Argentina, Laguna Otern Aike 1, Laguna Otern Aike 2 y Manantial, aunque en todos estos sectores dominan los nódulos de tamaño pequeño. 
Como se anticipó, para el análisis artefactual se seleccionaron los conjuntos arqueológicos líticos recuperados por medio de transectas y cuadrículas de recolección en el área central del IGCh. Como se observa en la Tabla 2 y Figura 6, la dominancia de la RGFO PKA en los conjuntos artefactuales es heterogénea. La abundancia de esta variedad de roca disminuye de norte a sur, siendo los espacios localizados en el extremo sur de la meso-transecta (Eas. Osasuna y Pali Aike) los que registran el menor aporte de RGFO PKA.

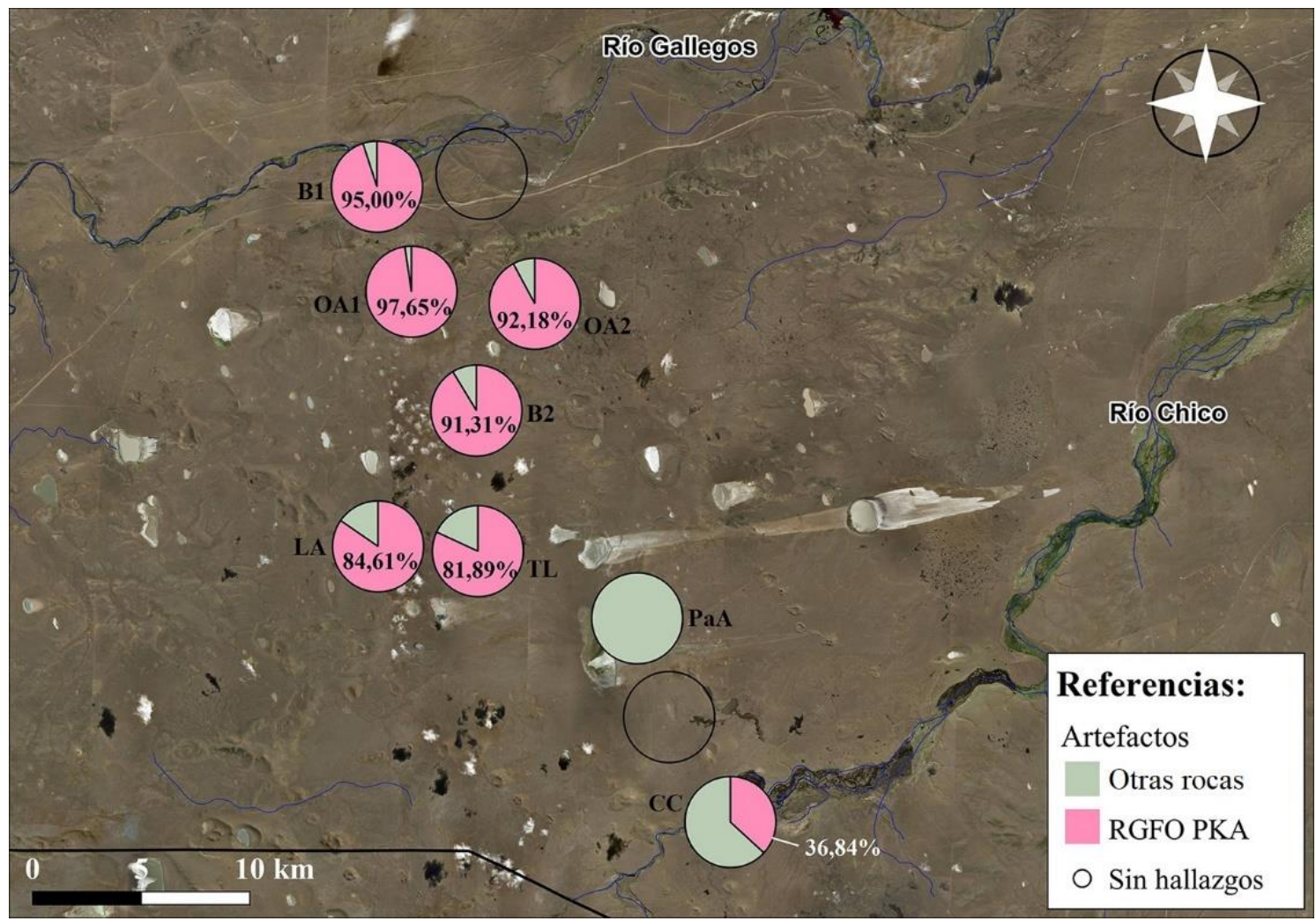

Figura 6. Proporción de artefactos confeccionados con RGFO PKA en los distintos sectores muestreados de la meso-transecta. Referencias: $\mathrm{B} 1=$ Bajo 1; OA1= Laguna Otern Aike 1; OA2= Laguna Otern Aike 2; B2= Bajo 2; LA= Laguna La Argentina; TL= M1 y M2 en Laguna Tres Lagunas; $\mathrm{PaA}=$ Laguna Pali Aike; $\mathrm{CC}=$ Cerro Cóndor.

En todos los conjuntos, los artefactos de RGFO PKA están dominados por los desechos de talla, aunque cabe destacar que mientras en los muestreos del área central de la meso-transecta el aporte de núcleos es mayor que el de los instrumentos, en los espacios localizados en los extremos de la meso-transecta (Bajo 1 y Cerro Cóndor) la tendencia se invierte (Tabla 2). Además, la comparación de las tres clases artefactuales (núcleos, desechos de talla e instrumentos) mediante el cálculo de probabilidad de Monte Carlo arrojó diferencias estadísticamente significativas ( $\mathrm{chi}^{2}=64,524 \mathrm{p}=3,3376 \mathrm{E}-09$, Monte Carlo=0,0001, 9999 permutaciones). Las diferencias se deben principalmente a los conjuntos artefactuales de Laguna Otern Aike 2, Bajo 2, Laguna La Argentina y Cerro Cóndor. Los residuos ajustados 
del test muestran que en Laguna Otern Aike 2 y Laguna La Argentina, con fuentes de RGFO PKA de rendimiento intermedio-alto y muy alto respectivamente, se registran más núcleos e instrumentos y menos desechos de talla de lo esperado por azar, mientras que en el Bajo 2, sector donde sólo se recuperaron nódulos de RGFO PKA fuera del tiempo de muestreo, se reconocen menos núcleos e instrumentos y más desechos de talla de lo esperado. Por su parte, Cerro Cóndor, sin disponibilidad de RGFO PKA en sus inmediaciones, registró menos desechos de talla y más instrumentos de los esperados por azar. Estas tendencias son coherentes con la disponibilidad natural de las RGFO PKA en la meso-transecta.

\begin{tabular}{|c|c|c|c|c|c|c|c|c|c|c|}
\hline Estancia & $\begin{array}{c}\text { Sector } \\
\text { muestreado }\end{array}$ & $\begin{array}{l}\text { Sup. } \\
\left(\mathrm{m}^{2}\right)\end{array}$ & $\begin{array}{c}\text { N } \\
\text { Art. }\end{array}$ & $\begin{array}{c}\text { N Art. } \\
\text { RGFO } \\
\text { PKA }\end{array}$ & $\mathrm{Nu}$ & DT & I & $\mathbf{N u} \%$ & DT $\%$ & $\mathrm{I} \%$ \\
\hline \multirow[b]{2}{*}{$1^{\circ}$ de Mayo } & Bajo 1 & 375 & 40 & 38 & 2 & 32 & 4 & 5,26 & 84,21 & 10,53 \\
\hline & $\begin{array}{c}\text { Transectas } \\
1 \text { a } 4\end{array}$ & 80000 & 0 & 0 & 0 & 0 & 0 & 0 & 0 & 0 \\
\hline \multirow{2}{*}{ Otern Aike } & $\begin{array}{c}\text { Laguna Otern } \\
\text { Aike } 1\end{array}$ & 5027 & 809 & 790 & 64 & 693 & 33 & 8,10 & 87,72 & 4,18 \\
\hline & $\begin{array}{c}\text { Laguna Otern } \\
\text { Aike } 2\end{array}$ & 26000 & 243 & 224 & 28 & 176 & 20 & 12,50 & 78,57 & 8,93 \\
\hline Don Raúl & Bajo 2 & 1336 & 495 & 452 & 28 & 415 & 9 & 6,19 & 91,82 & 1,99 \\
\hline $\begin{array}{c}\text { La } \\
\text { Argentina }\end{array}$ & $\begin{array}{l}\text { Laguna La } \\
\text { Argentina }\end{array}$ & 26000 & 65 & 55 & 11 & 35 & 9 & 20 & 63,64 & 16,36 \\
\hline $\begin{array}{c}\text { Tres } \\
\text { Lagunas }\end{array}$ & $\begin{array}{l}\text { M1 y M2 en } \\
\text { Laguna Tres } \\
\text { Lagunas }\end{array}$ & 200 & 718 & 588 & 46 & 510 & 32 & 7,82 & 86,74 & 5,44 \\
\hline \multirow{2}{*}{ Osasuna } & $\begin{array}{l}\text { Laguna Pali } \\
\text { Aike } \\
\end{array}$ & 4500 & 4 & 0 & 0 & 0 & 0 & 0 & 0 & 0 \\
\hline & $\begin{array}{c}\text { Transectas } 9, \\
10,11,21 \text { y } 22\end{array}$ & 81000 & 0 & 0 & 0 & 0 & 0 & 0 & 0 & 0 \\
\hline Pali Aike & Cerro Cóndor & 20008 & 19 & 7 & 1 & 4 & 2 & 14,29 & 57,14 & 28,57 \\
\hline
\end{tabular}

Tabla 2. Artefactos y superficies muestreadas por sector. Referencias: N Art. (Número total de artefactos), Nu (Núcleo), DT (Desecho de Talla) e I (Instrumento).

Los datos obtenidos a partir de la cuantificación del remanente de corteza de los núcleos $(\mathrm{n}=180)$ muestran variabilidad en la intensidad de explotación registrada a lo largo de la meso-transecta. En el área central, y en concordancia con la mayor disponibilidad natural de RGFO PKA, la explotación de nódulos de esta materia prima es variable, registrándose núcleos desde intensamente explotados (0\% de reserva de corteza) a muy poco explotados $(76 \%-<100 \%$ de reserva de corteza). En cambio, en los extremos de la meso-transecta (Bajo 1 y 
Cerro Cóndor), donde la disponibilidad de RGFO PKA es escasa a nula, sólo se registran núcleos muy explotados (1\% - $25 \%$ de reserva de corteza) (Figura 7a). Asimismo, algunos sectores del área central del IGCh registran mayor intensidad de explotación de núcleos que otras. Este es el caso de M1 y M2 en Laguna Tres Lagunas, que exhiben una mayor frecuencia de núcleos intensamente explotados $(13,04 \%)$ y muy explotados $(41,30 \%)$ con respecto a otros sectores de la localidad. Esto también se puede observar en Laguna Otern Aike 1 y Bajo 2, donde el rango de corteza correspondiente al intervalo de $76-<100 \%$ no se encuentra representado. Cabe destacar que en Laguna Otern Aike 1 y 2, Bajo 2 y Laguna La Argentina predominan los núcleos con evidencias de intensidad media $(39,06 \%$, $46,42 \%, 42,86 \%, 36,36 \%)$ y alta de explotación $(32,81 \%, 28,57 \%, 28,57 \%, 36,36 \%)$, respectivamente.

Entre los desechos de talla $(n=1865)$, y sobre la base de los remanentes de corteza, se observa diversidad en la intensidad de reducción de núcleos (Figura $7 b)$. En la mayoría de los sectores predominan los estadios avanzados de reducción, ya que se reconoce un mayor aporte de desechos sin corteza (Bajo 1 $=37,5 \%$, Laguna Otern Aike $1=41,27 \%$, Laguna Otern Aike $2=33,52 \%$, Bajo $2=$ $44,58 \%$, M1 y M2 en Laguna Tres Lagunas $=44,31 \%)$. Una excepción es el conjunto de Laguna La Argentina que se encuentra mayormente representado por estadios iniciales de talla $(76-100 \%$ de reserva de corteza $=25,71 \%$ y $51-75 \%$ de reserva de corteza $=25,71 \%)$. Desechos que pueden ser atribuidos a los primeros estadios de reducción (76-100 \% de reserva de corteza) se encuentran también en Laguna Otern Aike 2 (19,32 \%), M1 y M2 en Laguna Tres Lagunas (18,23 \%), Bajo 1 $(15,62 \%)$, Laguna Otern Aike 1 (15,58 \%) y Bajo 2 (11,32 \%). Por su parte, Cerro Cóndor, extremo sur de la meso-transecta y donde no se verificó la presencia natural de nódulos RGFO PKA, no registra desechos vinculados a los primeros estadios de reducción, aunque sí se encontraría representado el resto de las etapas ( 0 a $75 \%$ de reserva de corteza).

Los instrumentos $(n=109)$ con algún remanente de corteza se encuentran ampliamente representados en los conjuntos artefactuales de los distintos sectores (Laguna Otern Aike $1=45,45 \%$, Bajo $1=50,00 \%$, Laguna Otern Aike $2=60,00 \%$, M1 y M2 en Laguna Tres Lagunas 62,50 \%, Bajo $2=77,78$ \%, Laguna La Argentina = $81,81 \%$ y Cerro Cóndor $=100 \%$ ). Los instrumentos sin reserva de corteza alcanzan el 39,64 \% del total de la muestra y proceden de Laguna Otern Aike 1 (54,54 \%), Bajo 1 (50,00 \%), Laguna Otern Aike 2 (40,00 \%), M1 y M2 en Laguna Tres Lagunas $(37,5 \%)$, Bajo $2(22,22 \%)$ y Laguna La Argentina (18,18\%). En este último sector $(9,09 \%)$ y en M1 y M2 (3,12 \%) las formas base exhiben una muy alta cobertura cortical (76-100 \%). Valores altos de remanente de corteza en artefactos formatizados (51-75 \%) se registran en Bajo 2 (44,55\%) y en otros sectores, aunque en menor proporción (Figura 7c). 


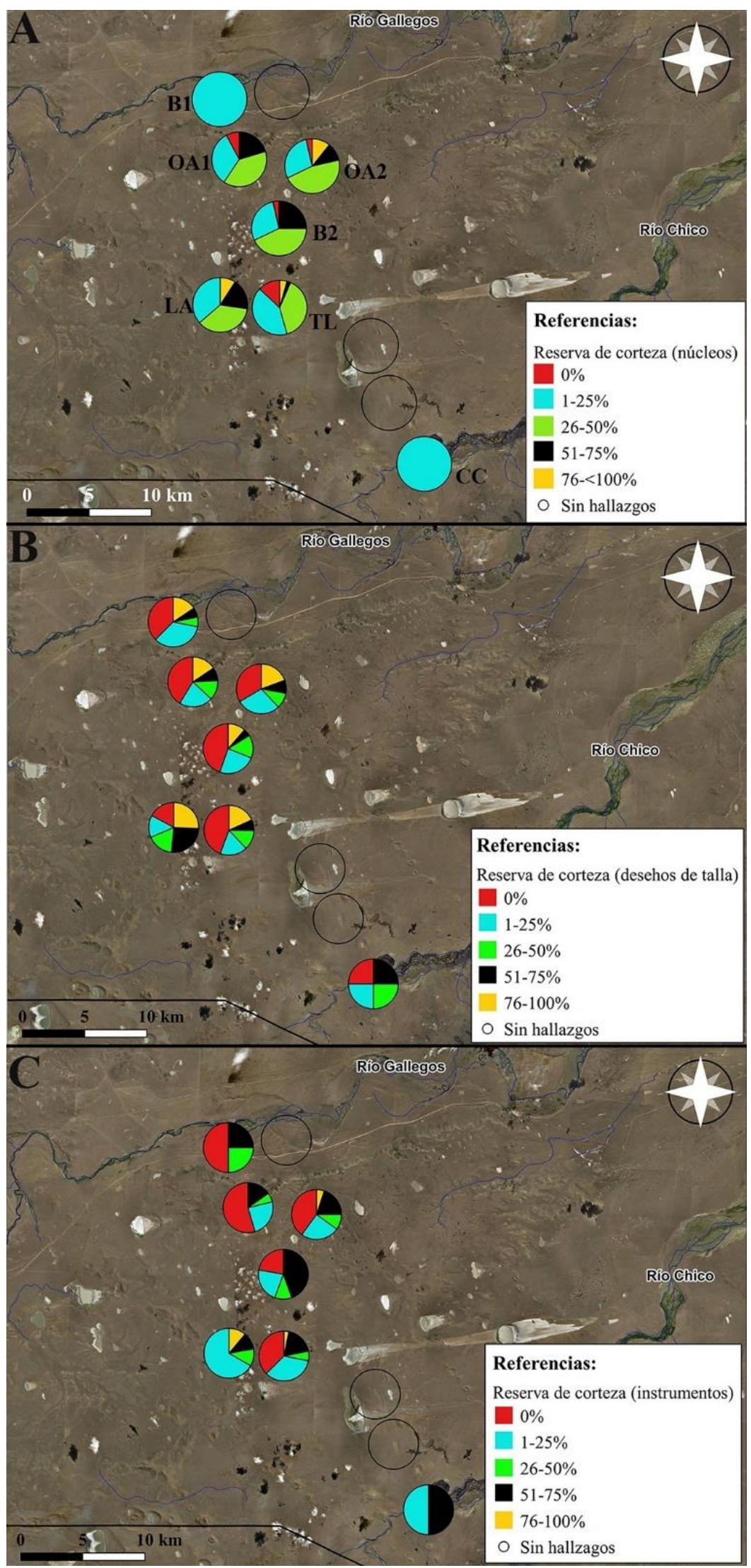

Figura 7. Porcentaje de reserva de corteza en artefactos confeccionados sobre RGFO PKA. Referencias: A (Núcleos); B (Desechos de Talla); C (Instrumentos); B1 = Bajo 1; OA1= Laguna Otern Aike 1; OA2= Laguna Otern Aike 2; B2= Bajo 2; LA= Laguna La Argentina; TL= M1 y M2 en Laguna Tres Lagunas; $\mathrm{CC}=$ Cerro Cóndor. 
En la muestra de núcleos dominan aquéllos de tamaños mediano-pequeños $(48,89 \%)$ y pequeños $(41,66 \%)$, y sólo en algunos sectores se recuperaron núcleos de dimensiones mayores, aunque en baja frecuencia (Figura 8A). Los núcleos grandes $(0,56 \%)$ se reconocieron únicamente en Laguna La Argentina (9,09\%), mientras que los mediano-grandes $(5,56 \%)$ en los sectores Bajo 2 (14,28\%), Laguna La Argentina (9,09 \%), Laguna Otern Aike 1 (4,69 \%) y M1 y M2 en Laguna Tres Lagunas (4,34 \%). Exceptuando el Bajo 2, el rango de tamaño de los núcleos representados en esos sectores parece estar en relación con los volúmenes de los nódulos recuperados en los muestreos de RGFO PKA. Los núcleos de tamaño mediano-pequeño $(60,71 \%$ ) y mediano-grande recolectados en Bajo 2 de Ea. Don Raúl no se condicen con el tamaño de los guijarros de tamaños pequeños recobrados fuera del tiempo de muestreo y a una distancia localizada entre $100 \mathrm{y}$ $1000 \mathrm{~m}$ de la depresión. Por otra parte, los núcleos de tamaños muy pequeños $(3,33 \%)$ se registraron únicamente en Laguna Otern Aike $2(17,86 \%)$ y Laguna Otern Aike 1 (1,56 \%). Cabe destacar que Cerro Cóndor, alejado de las fuentes de RGFO PKA, registró un núcleo de tamaño mediano-pequeño, aunque con una reserva de corteza en el rango de 1 y $25 \%$.

En la muestra general de desechos de talla predominan aquéllos con tamaños muy pequeños $(48,15 \%)$ y pequeños $(46,49 \%$ ) (Figura 8B). Los elementos de tamaños mediano-grandes $(4,99 \%)$ y mediano-pequeños $(0,38 \%)$ se registran en menor frecuencia. Los mediano-grandes se reconocen en todos los sectores con excepción de Cerro Cóndor, hallándose en mayor proporción en Laguna La Argentina (28,57 \%) y Laguna Otern Aike 2 (10,79 \%). En Cerro Cóndor, sólo se registraron desechos de talla de tamaños muy pequeños $(50,00 \%)$ y pequeños $(50,00 \%)$.

En el conjunto de instrumentos dominan aquéllos de tamaños pequeños (57,80 $\%)$ y mediano-pequeños (32,11 \%). Este cambio en la frecuencia con respecto a las dimensiones de los desechos de talla podría estar indicando una selección de soportes algo más grandes para la confección de instrumentos en RGFO PKA. Cabe destacar que las formas base de dimensiones mayores son las medianograndes (3,67 \%) y se registran en Bajo 2 (11,11 \%), Laguna La Argentina (11,11 \%), Laguna Otern Aike 2 (5,00 \%) y M1 y M2 en Laguna Tres Lagunas (3,12 \%) (Figura $8 C$ ). Los instrumentos recuperados en Cerro Cóndor son pequeños ( $n=1 ; 50,00 \%$ ) y mediano-pequeños ( $\mathrm{n}=1 ; 50,00 \%)$ y aquéllos recolectados en Bajo 1 son únicamente pequeños. 


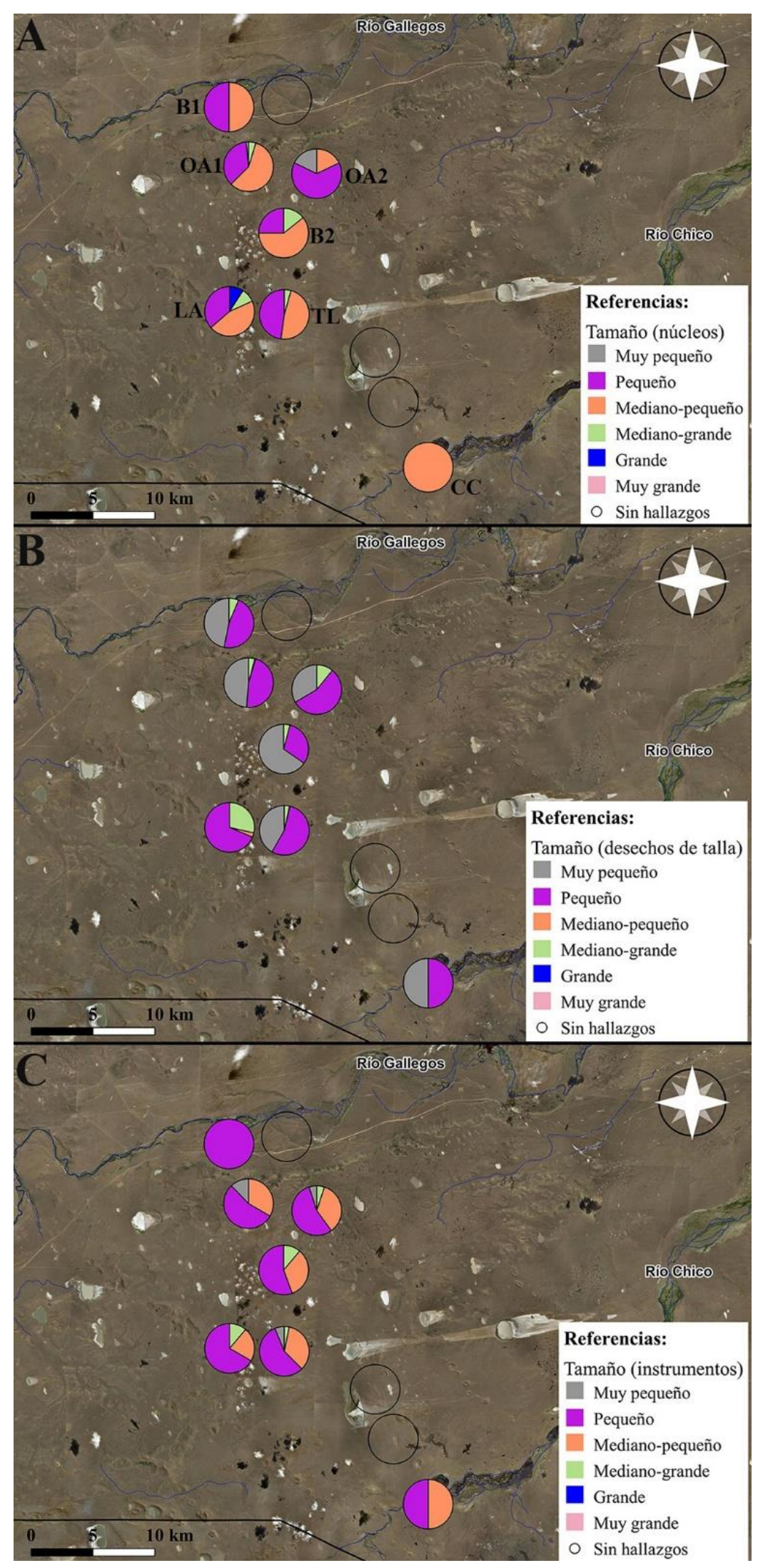

Figura 8. Porcentaje de tamaños de artefactos elaborados con RGFO PKA. Referencias: A (Núcleos); B (Desechos de Talla); C (Instrumentos); B1 = Bajo 1; OA1= Laguna Otern Aike 1; $\mathrm{OA} 2=$ Laguna Otern Aike 2; B2= Bajo 2; LA= Laguna La Argentina; TL= M1 y M2 en Laguna Tres Lagunas; $\mathrm{CC}=$ Cerro Cóndor. 
El conjunto artefactual registra poca variabilidad en el rango de tamaños y amplia representación de todos los intervalos de remanentes de corteza (0 a $100 \%$ ). La comparación de los conjuntos artefactuales en función de esta última variable mediante el cálculo de probabilidad de Monte Carlo arrojó diferencias estadísticamente significativas ( $\mathrm{chi}^{2}=58,201 \mathrm{p}=1,14 \mathrm{E}-04$, Monte Carlo=0,0001, 9999 permutaciones). A partir de la lectura de los residuos ajustados se observa que las diferencias se deben principalmente a que, en algunos sectores, tales como Laguna La Argentina y Cerro Cóndor, hay más artefactos con mayor cobertura cortical que los esperados por azar, mientras que en otros, el aporte de esta característica es menor, por el ejemplo para el rango 76-100 \% del Bajo 2 y rango 1-25 \% de M1 y M2 de Laguna Tres Lagunas. Los mismo ocurre con la comparación de tamaños donde el cálculo de probabilidad de Monte Carlo también muestra diferencias significativas entre los conjuntos ( $\mathrm{chi}^{2}=207,9$ p=3,0751E-31, Monte Carlo=0,0031, 9999 permutaciones). En términos generales, las diferencias se vinculan con la existencia de artefactos de tamaños mayores (Laguna La Argentina y Cerro Cóndor) o menores (Bajo 2) a los esperados.

\section{Discusión}

Los estudios sobre la disponibilidad y características de los nódulos de RGFO PKA obtenidos en fuentes secundarias del IGCh constituyen el punto de partida para evaluar la forma e intensidad de explotación en los conjuntos arqueológicos recuperados en los sectores muestreados de la meso-transecta. Los resultados obtenidos apuntan en gran medida a que la distancia a la fuente de aprovisionamiento de RGFO PKA incidió en las estrategias implementadas por los grupos humanos para su explotación.

Los relevamientos realizados en la meso-transecta permitieron detectar nódulos de RGFO PKA en formaciones geológicas correspondientes a diversos periodos (Mioceno, Pleistoceno y Holoceno), así como en sus cicatrices de erosión (e.g. Basalto Pali Aike en Ea. Don Raúl o Depósitos aluviales y coluviales indiferenciados en Laguna Otern Aike 2). Los resultados obtenidos a partir de los muestreos de materias primas indican que las RGFO PKA exhiben una disponibilidad variable en el área central del IGCh, lo que permite aislar espacios con y sin presencia de esta materia prima. La mayor abundancia de RGFO PKA ha sido registrada en el centro de la meso-transecta (Laguna Otern Aike 1, Laguna Otern Aike 2 y Laguna La Argentina). En cambio, los espacios localizados próximos a los ríos Gallegos y Chico (extremos norte y sur de la mesotransecta) muestran bajo rendimiento o ausencia de nódulos respectivamente. Un caso particular a considerar es Bajo 2 (Ea. Don Raúl) que registró muy bajo rendimiento de nódulos de RGFO PKA, ya que se reconocieron sólo tres ejemplares en un relevamiento más intensivo realizado por fuera del tiempo de muestreo y a distintas distancias del bajo.

Aunque la calidad para la talla (muy buena) y la cantidad de corteza (100\%) es semejante entre los nódulos de las fuentes relevadas, se registran variaciones en la distribución de tamaños (longitud del eje mayor, volúmenes) con un claro incremento 
de norte a sur. No obstante, en la muestra general de nódulos dominan aquéllos de tamaños reducidos. Con respecto a la morfología, cabe destacar que en todos los sectores se recuperó gran cantidad de nódulos compactos y sin plataformas naturales aptas para la talla (Shelley 1993), lo que está en concordancia con la profusa evidencia de la aplicación de la técnica bipolar registrada sobre RGFO PKA y la identificación de yunques en el área (Figura 9) (Borrazzo et al. 2019; Borrazzo y Cirigliano 2020). Es significativo señalar que los tamaños reducidos y las morfologías compactas de los nódulos podrían influir en la forma de transportar esta materia prima, favoreciendo el acarreo mayormente de guijarros enteros desde las áreas de captación e introduciendo nuevas expectativas arqueológicas para los espacios más alejados a las fuentes $(v$. Franco 2014).

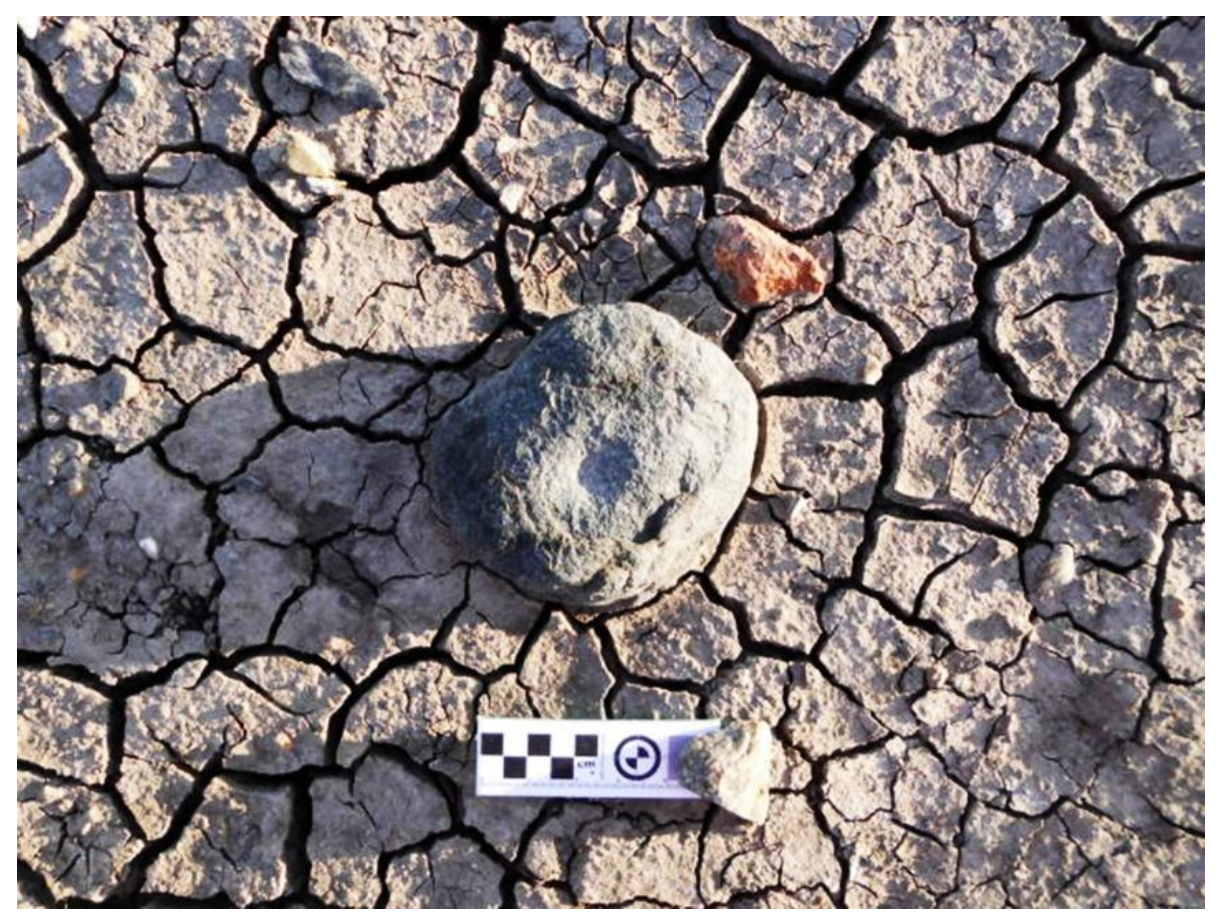

Figura 9. Yunque en Laguna Tres Lagunas.

Los cazadores-recolectores que ocuparon el área central del IGCh hicieron uso de las fuentes secundarias de RGFO PKA, explotaron preferencialmente esas materias primas en detrimento de otras en sus proximidades y transportaron posiblemente nódulos enteros a espacios cercanos naturalmente desprovistos de ellos. En líneas generales, los conjuntos arqueológicos de la meso-transecta registran poca variabilidad en los tamaños y alta proporción de artefactos con reserva de corteza, lo que se vincularía con las características de los nódulos disponibles naturalmente ( $v$. Bradbury y Carr 1995), pero también con su abundancia local (radio de hasta $40 \mathrm{~km}$ sensu Meltzer 1989) y su facilidad de acceso (sensu Nami 1992).

Como sugiere el modelo de adquisición directa (Renfrew 1977), la distancia a la fuente de aprovisionamiento incidió en la frecuencia de artefactos de RGFO PKA descartados en la meso-transecta. La disminución en el aporte de estas rocas en los conjuntos artefactuales queda reflejada en los espacios analizados al sur de M1 y M2 
(Ea. Tres Lagunas), aunque esto deberá ser investigado con mayor profundidad debido al pequeño tamaño de las muestras allí recuperadas. Cabe recordar que Nami trabajó previamente el sector de Laguna Pali Aike, donde recogió gran cantidad de materiales arqueológicos (1999, 2009; entre otros). En ese sentido, la tendencia observada en Pali Aike (cuatro artefactos No RGFO PKA) debe ser cuestionada por tratarse muy probablemente de una muestra sesgada. No obstante, la falta de nódulos de RGFO PKA en ese sector del espacio y el menor aporte de RGFO PKA en el conjunto de Cerro Cóndor con respecto al resto de los sectores muestreados, sugiere posiblemente valores de decrecimiento de esa materia prima en Pali Aike, lo que deberá ser contrastado a futuro con nuevos trabajos de campo o el análisis de colecciones.

La menor frecuencia de artefactos confeccionados sobre RGFO PKA en Cerro Cóndor se condice con las expectativas de adquisición directa de materia prima. No obstante, no se reconoce una tendencia en la disminución de tamaños o reserva de corteza en artefactos. Este hecho se podría vincular con la selección de nódulos para su transporte ( $c f$. Civalero y Franco 2003; Franco 2014) ${ }^{6}$. Especialmente, el conjunto de Cerro Cóndor registra una mayoría de artefactos de RGFO PKA con reserva de corteza ( $n=6 ; 85,71 \%)$ en distinta proporción (1-25 \%, 26-50 \% y 51-75 \%). Allí se reconoció un núcleo de tamaño mediano-pequeño, aunque con una reserva de corteza en el rango de 1 y $25 \%$ que posiblemente indique una alta explotación del mismo. Algo semejante pudo ocurrir en Bajo 2, donde la frecuencia y dimensiones de los núcleos recuperados, así como el alto aporte de ejemplares con reserva de corteza (aunque en menor proporción de lo esperado en el rango de los 76-100 \%), sugiere la implementación de una estrategia de transporte de nódulos de tamaños mayores a los inmediatamente disponibles desde algún lugar algo más alejado a la depresión.

Se reconocen diferencias en la intensidad de explotación de RGFO PKA que, además de las distancias a las fuentes de aprovisionamiento lítico, se relacionan con el uso diferencial de los espacios vinculado a la existencia o falta de otros recursos (e.g. agua, reparo) que podrían incrementar o reducir su probabilidad de uso (Ebert y Kohler 1988). Por ejemplo, sectores con rendimiento de RGFO PKA alto (e.g. Laguna Otern Aike 1) y relativamente bajo (e.g. M1 y M2 en Laguna Tres Lagunas) presentan una mayor densidad artefactual y de piezas sin reserva de corteza, lo que podría estar asociado con una mayor inversión de tiempo en la confección de artefactos y en el uso de esos espacios ( $c f$. Borrazzo y Cirigliano 2020). En cambio, Laguna La Argentina, pese a la muy alta oferta de RGFO PKA disponible, registra baja densidad artefactual y gran cantidad de artefactos con alta cobertura cortical que podría asociarse a una menor inversión de tiempo en la manufactura y duración de las ocupaciones.

En este punto es importante señalar que Laguna La Argentina y Laguna Tres Lagunas se encuentran a $c a .500 \mathrm{~m}$ de distancia, siendo el último de los sectores más propicio para la ocupación por ofrecer más reparo de los vientos predominantes del Oeste-Sudoeste. Sobre la base de la información arqueológica y los muestreos de materias primas, se plantea la posible complementariedad de esos dos sectores. En este 
sentido, se destaca la gran cantidad de ecofactos (nódulos de RGFO PKA) que fueron recuperados en Laguna Tres Lagunas en asociación a los conjuntos artefactuales que podrían proceder no sólo de este bajo, sino también de otros espacios próximos y más productivos en términos de rendimiento de la variedad PKA. Muestreos de materias primas más intensivos (i.e. para evaluar variaciones en la disponibilidad en una escala espacial más reducida) deben ser realizados en otras márgenes de Laguna Tres Lagunas y sus espacios adyacentes hacia el Este para corroborar la hipótesis de complementariedad planteada entre ambos bajos sin salida.

\section{Conclusión}

La información entregada por este trabajo apoya la propuesta de que el sector central del CVPA fue una gran área de captación de RGFO PKA, al menos durante el Holoceno tardío (Charlin y D'Orazio 2010; Charlin y Pallo 2013). Las observaciones efectuadas en el registro lítico de la meso-transecta son las esperadas para el modelo de adquisición directa de RGFO PKA. Sin embargo, nuestra investigación señala la existencia de variaciones significativas en la disponibilidad de las RGFO PKA a escala más pequeña, las cuales fueron incorporadas activamente por las sociedades cazadoras recolectoras en sus decisiones sobre la manufactura lítica y el uso del espacio. De acuerdo a nuestras observaciones, la dispersión natural de RGFO PKA no alcanzaría espacios localizados al sur de la Laguna Pali Aike (Ea. Osasuna), donde sí se han recuperado artefactos elaborados con RGFO PKA cuyas características son compatibles con la adquisición directa de nódulos en las fuentes. Por otra parte, cabe destacar que se reconocen variaciones que van más allá de la disponibilidad del recurso lítico y que se encuentran ligadas a las formas de ocupar los espacios (más intensa y menos intensa) en función de la potencialidad de cada lugar.

Nuestros resultados sugieren que los trabajos de campo abocados a ampliar el conocimiento sobre la base regional de la RGFO PKA en el IGCh y espacios aledaños necesariamente deberán evaluar variaciones en la disponibilidad en una escala espacial más reducida, así como examinar posibles cambios a lo largo del tiempo que pudieron influir su disponibilidad natural. Con relación a esto último, cabe recordar que los relevamientos ejecutados permitieron reconocer nódulos de RGFO PKA en formaciones geológicas correspondientes a diferentes periodos o en sus cicatrices de erosión, lo que advierte sobre la importancia de la dinámica geológica y geomorfológica en la creación y sepultamiento de fuentes de aprovisionamiento (e.g. Franco 1998), y lleva a considerar la posibilidad de cambios en la disponibilidad de RGFO PKA a través del tiempo.

Las tendencias registradas indican la necesidad de explorar espacios adyacentes a la meso-transecta y revisar colecciones artefactuales procedentes del sector norte y sur del CVPA. Esto permitirá evaluar las hipótesis propuestas al respecto de las elecciones y los criterios que guiaron la toma de decisiones de cazadores-recolectores vinculadas con la explotación y circulación de las RGFO PKA. En ese sentido, habilitará a 
continuar indagando acerca del nodo poblacional del CVPA y sus conexiones con otros espacios alejados ( $c f$. Balirán 2021; Belardi et al. 2020; entre otros). Esto, sumado a la búsqueda de nuevos sitios arqueológicos nos permitirá tener un panorama más completo sobre el uso de la RGFO PKA a escala regional.

Agradecimientos: La investigación fue financiada por Proyectos ANPCyT (PICT 20142061, PICT 2019-02106, PICT 2019-01356) y CONICET. Agradecemos muy especialmente a Myrna Clark y familia Fernández-Clark, Shirley Stewart y Guillermo Clark, Ariel Hamilton, Horacio Clark, Lili Zahn y Federico Rodríguez Zahn, y Federico Kennard. También agradecemos a Luis Borrero, Marcelo Cardillo y a nuestras compañeras de equipo (Judith Charlin, Lorena L'Heureux, Liliana Manzi, Ivana Ozán y Cecilia Pallo) por su aporte valioso en gabinete y/o trabajos de campo. Por último, agradecemos a los evaluadores, cuyos comentarios y sugerencias contribuyeron a mejorar el trabajo.

\section{Notas}

${ }^{1}$ Cabe mencionar que los nódulos de RGFO PKA más grandes fueron recuperados en Laguna Potrok Aike. En ese sector, el volumen máximo registrado fue 1433,82 cm (Cirigliano y Charlin 2019).

2 Las investigaciones realizadas con anterioridad a los estudios geoquímicos y petrográficos señalaban al basalto como la roca más seleccionada para la elaboración de artefactos (e.g. Gómez Otero 1986-87; Sanguinetti de Bórmida 1976).

3 Aunque el sector sur del CVPA registra la señal arqueológica más intensa a nivel regional (Barberena 2008; Charlin 2009).

4 Durante las prospecciones realizadas en la Formación $10\left(80.000 \mathrm{~m}^{2}\right)$ se reconocieron gran cantidad de sectores erosionados con abundante disponibilidad de rocas volcánicas de colores claros junto con escasas RGFO, aunque no se identificó la variedad PKA.

5 Se reconocieron artefactos líticos en proximidades al Manantial que no fueron recolectados debido al escaso tiempo disponible para realizar la tarea.

${ }^{6}$ Es importante destacar que un registro arqueológico semejante podría ser esperado en caso de existir la posibilidad de obtener nódulos de RGFO PKA de manera muy escasa e impredecible. Dado que esta forma de encuentro facilitaría la explotación oportunística del recurso (sensu Nelson 1991), estudios más intensivos que involucren trabajos de campo deben ser realizados para ajustar las interpretaciones y reducir problemas de equifinalidad.

\section{Bibliografía citada}

Alberti, J.

2012 Fuentes de rocas y uso de materias primas líticas en Bahía Final 6, costa norte del golfo San Matías (Río Negro, Argentina). Intersecciones en Antropología 13: 237-249. 
Apolinaire, E.

2018 Caracterización de los depósitos secundarios de rodados fluviales del río Uruguay inferior: su aplicación en contextos arqueológicos de las llanuras interiores del nordeste argentino. Boletim do Museu Paraense Emílio Goeldi. Ciências Humanas 13 (2): 443-457.

Aschero, C. A.

1975 Ensayo para una clasificación morfológica de artefactos líticos aplicada a estudios tipológicos comparativos. Informe al CONICET. Buenos Aires. Ms.

1983 Ensayo para una clasificación morfológica de artefactos líticos aplicada a estudios tipológicos comparativos. Revisión. Cátedra de Ergología y Tecnología (FFyLUBA). Buenos Aires. Ms.

Balirán, C.

2021 El pasado pisado. Investigaciones arqueológicas en tecnología y tafonomía líticas de los cazadores recolectores de Sierra Baguales, Sudoeste de Santa Cruz, Argentina. IMHICIHUCONICET, Buenos Aires.

Barberena, R.

2008 Arqueología y biogeografía humana en Patagonia Meridional. Colección Tesis Doctorales. Sociedad Argentina de Antropología, Buenos Aires.

Belardi, J.B., F. Carballo Marina y L.A. Borrero

2020 Circulación humana durante el Holoceno tardío en el bosque y ecotono bosqueestepa: el curso inferior del río Penitente (suroeste de Santa Cruz). Revista de Museo de Antropología 13 (3): 321-334.

Belardi, J.B., G. Cassiodoro, R. Goñi, M. Glascock y A. Súnico

2015 Siltstone from Southern Patagonia: its source and archaeological artifact distribution in Santa Cruz Province, Argentina. Geoarchaeology 30 (3), 223-237.

Binford, L.R.

1979 Organization and formation processes: looking at curated technologies. Journal of Anthropological Research 35: 255-273.

Bird, J.

1988 Travels and Archaeology in South Chile. University of Iowa Press, Iowa.

Bobillo, F. M. y S. Hocsman

2015 Mucho más que solo aprovisionamiento lítico: actividades en canteras y prácticas sociales en las fuentes de Pampa Oeste, Quebrada Seca y Punta de la Peña (Antofagasta de la Sierra, Catamarca. Revista del Museo de Antropología 8 (1): 23-44. 
Borrazzo, K. y N. Cirigliano

2020 La organización de la tecnología lítica en el interfluvio Gallegos-Chico (Santa Cruz, Argentina): una meso-transecta a los $\mathrm{O} 70^{\circ}$ en el campo volcánico Pali Aike. Relaciones de la Sociedad Argentina de Antropología XLV (1): 175-199.

Borrazzo, K., G.L. L'Heureux, N. Cirigliano, M.C. Pallo, I. Ozán, L. Manzi y J. Charlin 2019 Prospecciones en el interfluvio Gallegos-Chico (Santa Cruz, Argentina): nuevos datos arqueológicos. Arqueología de la Patagonia: el pasado en las arenas (ed. por J. Gómez Otero, A. Svobova y A. Banegas), pp. 271-282. Sociedad Argentina de Antropología, Buenos Aires.

Borrazzo, K., F. Morello, L.A. Borrero, M. D'Orazio, M.C. Etchichury, M. Massone y H. De Angelis

2015 Caracterización de las materias primas líticas de Chorrillo Miraflores y su distribución arqueológica en el extremo meridional de Fuego-Patagonia. Intersecciones en Antropología - Volumen especial 2: 155-167.

Bradbury, A.P. y P.J. Carr

1995 Flake typologies and alternative approaches: an experimental assessment. Lithic Technology 20 (2):100-115.

Catella, L., G. Barrientos y F. Oliva

2017 La identificación del uso de fuentes secundarias de materiales líticos asistida por SIG: el Arroyo Chasicó (Argentina) como caso de estudio. Estudios Geológicos 73 (1), doi: http://dx.doi.org/10.3989/egeol.42720.441

Charlin, J.

2005 Utilización de materias primas líticas en el campo volcánico Pali Aike (Pcia. de Santa Cruz, Argentina). Una primera aproximación a partir del análisis de núcleos. Werken 7:39-55.

2009 Estrategias de aprovisionamiento y utilización de las materias primas líticas en el campo volcánico Pali Aike (Prov. Santa Cruz, Argentina). BAR International Series 1901. Archaeopress, Oxford.

Charlin, J., L.A. Borrero y M.C. Pallo

2011 Ocupaciones humanas en el área noroccidental del río Gallegos. Bosques, montañas y cazadores: investigaciones arqueológicas en Patagonia meridional (ed. por L.A. Borrero y K. Borrazzo), pp. 179-210. Dunken, Buenos Aires.

Charlin, J. y M. D’Orazio

2010 Fuentes primarias vs. secundarias de aprovisionamiento lítico: una comparación geoquímica (Pali Aike, Santa Cruz). La Arqueometría en Argentina y Latinoamérica (ed. por S. Bertolino, G.R. Cattaneo y A.D. Izeta), pp.153-158. Editorial 
de la Facultad de Filosofía y Humanidades, Universidad Nacional de Córdoba, Córdoba.

2015 Disponibilidad de materias primas líticas en la costa nororiental del estrecho de Magallanes (Chile). Magallania 43(2): 133-154.

\section{Charlin, J., N. Franco y M. D'Orazio}

2010 El Campo Volcánico Pali Aike y las fuentes potenciales de aprovisionamiento lítico: primeros resultados geoquímicos. Arqueología de Pali Aike y Cabo Vírgenes (Santa Cruz, Argentina) (ed. por L.A. Borrero y J. Charlin), pp. 31-54. IMHICIHU-CONICET, Buenos Aires.

Charlin, J. y M.C. Pallo

2013 Disponibilidad de materias primas líticas y uso del espacio en el interfluvio Gallegos-Chico (Pali Aike, Santa Cruz). Tendencias teórico-metodológicas y casos de estudio en la arqueología de Patagonia (ed. por A.F. Zangrando, R. Barberena, A. Gil, G. Neme, M. Giardina, L. Luna, C. Otaola, S. Paulides, L. Salgán y A. Tivoli), pp. 307-316. Museo de Historia Natural de San Rafael, INAPL y SAA, San Rafael.

2015 Disponibilidad de rocas y costos de aprovisionamiento en el extremo austral de Patagonia meridional: integración de resultados en una escala regional. Intersecciones en Antropología 16(3): 125-138.

\section{Cirigliano, N.A. y J. E. Charlin}

2019 Rocas de grano fino oscuras tipo Potrok Aike: una evaluación sobre su disponibilidad a escala temporal. XX Congreso Nacional de Arqueología Argentina, pp. 344-346. IDACOR, Córdoba, Argentina.

Cirigliano, N., M.C. Pallo y J. Charlin

2020 Explotación y circulación de rocas de grano fino oscuras tipo Potrok Aike: el análisis de dos conjuntos líticos de Patagonia extremo sur continental (Argentina). Revista del Museo de Antropología 13(1): 197-202.

Civalero, M.T. y N.V. Franco

2003 Early human occupations in western Santa Cruz province, Southernmost South America. Quaternary International 109-110: 77-86.

Clarkson, C.

2008 Changing Reduction Intensity, Settlement and Subsistence in Wardaman Country, Northern Australia. Lithic Technology: Measures of Production, Use, and Curation (ed. por W. Andrefsky), pp. 286-316. Cambridge University Press, Cambridge.

Cortegoso, V., L.Yebra, V. Durán, R. Barberena, G. Lucero, L. Cornejo, M. Giesso, B. Macdonald y M. Glascock

2020 Obsidian sources from the southern Andean highlands (Laguna del Diamante, Argentina and Chile): geochemical insights on geological complexity and human 
biogeography. Archaeological and Anthropological Sciences 12, https://doi.org/10.1007/s12520-019-01009-w

D'Orazio, M., S. Agostini, F. Mazzarini, F. Innocenti, P. Manetti, M.J. Haller y A. Lahsen

2000 The Pali Aike volcanic field, Patagonia: slab-window magmatism near the tip of South America. Tectonophysics 321: 407-427.

Ebert, J. y T. Kohler

1988 The theoretical basis of archaeological predictive modeling and a consideration of appropriate data collection methods. Quantifying the present and predicting the past: theory, method and application of archaeological predictive modelling (ed. por W. J. Judge y L. Sebastian), pp. 97-123. U. S. Department of the Interior, Bureau of Land Management, Denver.

Ericson, J. E.

1984 Towards the Analysis of Lithic Production Systems. Prehistoric Quarries and Lithic Production (ed. por J.E. Ericson y B.A. Purdy), pp. 1-9. Cambridge University Press, Cambridge.

Franco, N.V.

1998 La utilización de recursos líticos en Magallania. Arqueología de la Patagonia Meridional (comp. Por L. Borrero), pp. 29-51. Ediciones Búsqueda de Ayllu, Entre ríos.

2002 Estrategias de utilización de recursos líticos en la cuenca superior del río Santa Cruz (Argentina). Tesis doctoral. Facultad de Filosofía y Letras, Universidad de Buenos Aires. Buenos Aires, Ms.

2014 Lithic Artifacts and the Information about Human Utilization of Large Areas. Artefactos Líticos, Movilidad y Funcionalidad de Sitios: Problemas y Perspectiva (ed. por P. Escola y S. Hocsman), pp. 116 - 127. BAR - South American Archaeology Series, Oxford.

Franco N.V. y L.A. Borrero

1999 Metodología de análisis de la estructura regional de recursos líticos. En los tres reinos: prácticas de recolección en el cono sur (ed. por C. Aschero, M. Korstanje y P. Vuoto), pp. 27-37. Instituto de Arqueología y Museo (FCN e IML - UNT). Ediciones Magna Publicaciones, San Miguel de Tucumán.

Gómez Otero, J.

1986-87 Investigaciones arqueológicas en el Alero Potrok Aike, provincia de Santa Cruz. Una revisión de los Períodos IV y V de Bird. Relaciones de la Sociedad Argentina de Antropología XVII (1): 173-200. 
Graham, D. y N. Midgley

2000 Graphical Representation of Particle Shape using Triangular Diagrams: An Excel Spreadsheet Method. Earth Surface Process and Landforms 25: 1473-1477.

2003 Tri-plot: Ternary diagram plotting de la Universidad de Loughborough. https://www.lboro.ac.uk/microsites/research/physgeog/tri-plot/in dex.html

Hammer, Ø., D. A. T.Harper y P. D. Ryan

2001 PAST: Paleontological Statistics Software Package for Education and Data Analysis. Palaeontologia Electronica 4, 9 p.

http://palaeo-electronica.org/2001_1/past/issue1_01.htm

L'Heureux, G. L., K. Borrazzo, J. Charlin, L. Manzi, N. Cirigliano, M. C. Pallo e I. Ozán 2020 Arqueología del valle del río Chico e interfluvio Gallegos-Chico (Campo Volcánico Pali Aike, Argentina). Actualización de las Investigaciones en Patrimonio Cultural realizadas en Santa Cruz editado por Dirección de Patrimonio de Santa Cruz. Río Gallegos.

L'Heureux, G.L., K. Borrazzo y J. Charlin

2021 Cronología de las ocupaciones humanas en el campo volcánico Pali Aike: aportes desde el interfluvio Gallegos-Chico y valle medio del río Chico (Santa Cruz, Argentina). Ms.

Meltzer, D.

1989 Was Stone Exchange Among Eastern North American Paleoindians? Eastern Paleoindian lithic resource use (ed. por C. Ellis y J. Lothrop), pp. 11-39. Westview Press, Boulder.

Morello, F.

2005 Tecnología y métodos para el desbaste de lascas en el norte de Tierra del Fuego: los núcleos del sitio cabo San Vicente. Magallania 33 (2): 29-56.

Nami, H.G.

1992 El subsistema tecnológico de confección de instrumentos líticos y explotación de los recursos del ambiente: Una nueva vía de aproximación. Shincal 2: 33-53.

1994 Paleoindio, cazadores-recolectores y tecnología lítica en el extremo sur de Sudamérica continental. Arqueología de Cazadores-Recolectores. Limites, Casos y Aperturas (ed. por J.L. Lanata y L.A. Borrero), pp. 89-103. Arqueología Contemporánea 5, Buenos Aires.

1999 Arqueología en la localidad arqueológica de Pali Aike, cuenca del Río Chico (Provincia de Santa Cruz, Argentina). Praehistoria 3: 189-218.

2000 Investigaciones actualísticas y piedra tallada. I: Criterios experimentales para identificar lascas de talla bipolar: Su aplicación en la interpretación de artefactos arqueológicos de los extremos norte y sur de la Patagonia. III Congreso Argentino de Americanistas, pp. 229-270. Sociedad Argentina de Americanistas, Liga Naval Argentina, Buenos Aires. 
2009 Avances de las investigaciones arqueológicas en la localidad arqueológica de Pali Aike, extremo sur de la provincia de Santa Cruz. Estado actual de las investigaciones realizadas sobre patrimonio cultural en Santa Cruz, pp. 235-241. Subsecretaría de Cultura de Santa Cruz, Río Gallegos.

Nelson, M.

1991 The Study of Technological Organization. Archaeological Method and Theory 3: 57100.

Odell, G.

1996 Economizing behavior and the concept of "curation". Stone tools: theoretical insights into human prehistory (ed. por G. Odell), pp. 51-80. Plenum Press, New York.

Oliva, G., L. González, P. Rial y E. Livrachi

2001 Áreas Ecológicas de Santa Cruz y Tierra del Fuego. Ganadería ovina sustentable en la Patagonia Austral. Tecnología de manejo extensivo (ed. por P. Borrelli y G. Oliva), pp. 4182. INTA, Río Gallegos.

Panza, J. y L. Sacomani

2015 Hojas Geológicas 5169-III y 5169-IV Río Gallegos y Cabo Buen Tiempo. Provincia de Santa Cruz. Boletín 413. SEGEMAR e IGRM.

Phillipps, R. S. y S. J. Holdaway

2016 Estimating core number in assemblages: core movement and mobility during the Holocene of the Fayum, Egypt. Journal of Archaeological Method and Theory 23: 520540 .

Renfrew, C.

1977 Alternative models for exchange and spatial distribution. Exchange Systems in Prehistory (ed. por T. Earle y J. Ericson), pp. 71-90. Academic Press, New York.

Sanguinetti de Bórmida, A.

1976 Excavaciones prehistóricas en la Cueva Las Buitreras, Santa Cruz. Relaciones de la Sociedad Argentina de Antropología X: 271-292.

Skarbun, F.

2011 La organización tecnológica en grupos cazadores recolectores desde las ocupaciones del Pleistoceno final al Holoceno tardío en la Meseta Central de Santa Cruz. BAR International Series. Archaeopress, Oxford.

Skewes, M.A.

1978 Geología, petrología, quimismo y origen de los volcanes del área de Pali Aike, Magallanes, Chile. Anales del Instituto de la Patagonia 9: 95-106. 


\section{Shelly, P.}

1993 A geoarchaeological approach to the analysis of secondary lithic deposits. Geoarchaeology: An International Journal 8(1): 59-72.

\section{Shiner, J.}

2004 Place as occupational histories: towards an understanding of deflated surface artifact distributions in the West Darling, New South Wales, Australia.

Tesis Doctoral. Department of Anthropology, University of Auckland, Auckland, Ms.

Sneed E.D. y R.L. Folk

1958 Pebbles in the lower Colorado River, Texas, a study of particle morphogenesis. Journal of Geology 66(2): 114-150.

Stern, C.

2018 Obsidian sources and distribution in Patagonia, southernmost South America. Quaternary International 468 (A): 190-205. 